\title{
Optimal Resource Allocation for Pervasive Health Monitoring Systems with Body Sensor Networks
}

\author{
Yifeng He, Member, IEEE, Wenwu Zhu, Fellow, IEEE, and Ling Guan, Fellow, IEEE
}

\begin{abstract}
Pervasive health monitoring is an eHealth service, which plays an important role in prevention and early detection of diseases. There are two major challenges in pervasive health monitoring systems with Body Sensor Networks (BSNs). The first challenge is the sustainable power supply for BSNs. The second challenge is Quality of Service (QoS) guarantee for the delivery of data streams. In this paper, we optimize the resource allocations to provide a sustainable and high-quality service in health monitoring systems. Specifically, we formulate and solve two resource optimization problems, respectively. In the first optimization problem, steady-rate optimization problem, we optimize the source rate at each sensor to minimize the rate fluctuation with respect to the average sustainable rate, subject to the requirement of uninterrupted service. The first optimization problem is solved by a proposed analytical solution. The second optimization problem is formulated based on the optimal source rates of the sensors obtained in the steady-rate optimization problem. In the second optimization problem, we jointly optimize the transmission power and the transmission rate at each aggregator to provide QoS guarantee to data delivery. The second optimization problem is converted into a convex optimization problem, which is then solved efficiently. In the simulations, we demonstrate that the proposed optimized scheme enables the pervasive health monitoring system to provide a sustainable service with guaranteed low delay and low Packet Loss Rate (PLR) to subscribers.
\end{abstract}

Index Terms-Body sensor networks, pervasive health monitoring, optimal resource allocation, quality of service (QoS), sustainable power supply, energy harvesting, convex optimization.

\section{INTRODUCTION}

$T^{1}$ HE worldwide population aged above 65 is expected to increase from 6.9 percent in 2000 to 12.0 percent in 2030 [1]. The ageing population will lead to increased healthcare cost as care for the elderly is much more expensive than that of other age groups. Electronic Health (eHealth), which integrates information processing and communications technologies into traditional medical services, emerges as a promising approach to improve healthcare efficiency. Pervasive health monitoring is an eHealth service, which plays an important role in prevention and early detection of diseases [2], [3], [4], [5].

A pervasive health monitoring system with Body Sensor Networks (BSNs) [6] is illustrated in Fig. 1. The eHealth service provider provides a health monitoring service to customers. The person who subscribes the service from the eHealth provider is called a subscriber. Each subscriber wears a BSN on his or her body. A BSN is a body-area wireless sensor network, which consists of multiple wireless body sensors and an aggregator. A body sensor can be worn on the body or implanted inside the body. An

- Y. He and L. Guan are with the Department of Electrical and Computer Engineering, Ryerson University, 350 Victoria Street, Toronto, ON M5B 2K3, Canada. E-mail: \{yhe, lguan\}@ee.ryerson.ca.

- W. Zhu is with the Internet Media Computing Group, Microsoft Research Asia, 5/F, Beijing Sigma Center, No. 49 Zhichun Road, Hai Dian District, Beijing 100190, China. E-mail: wenwuzhu@microsoft.com.

Manuscript received 12 Feb. 2010; revised 3 Nov. 2010; accepted 2 Dec. 2010; published online 20 Apr. 2011.

For information on obtaining reprints of this article, please send e-mail to: tmc@computer.org, and reference IEEECS Log Number TMC-2010-02-0070. Digital Object Identifier no. 10.1109/TMC.2011.83. aggregator can be a PDA or a cell phone. The body sensors continuously monitor the patient's vital signs, and then transmit them to the aggregator via wireless channels. The eHealth provider provides the service in multiple eHealth locations, such as the clinic, the mall, and the residence. An eHealth location is a coverage area of Wireless Metropolitan Access Network (WMAN). The subscribers in an eHealth location can transmit the data to the Base Station (BS), which further forward the data to the medical server over the Internet. As long as the subscribers are in one of the eHealth locations, they will always be monitored even in the presence of mobility.

There are two major challenges in pervasive health monitoring systems with body sensor networks. The first challenge is the sustainable power supply for body sensor networks [7]. The body sensors are powered by battery. Battery replacement is impossible for the sensors implanted inside the human body. The most promising approach to deal with the energy supply problem for BSNs is energy harvesting or energy scavenging [6], [8], [9]. In this approach, the sensor has an energy harvesting device that collects energy from ambient sources such as vibration and motion, light, and heat. However, the energy recharging rate is typically slow and time-varying. Therefore, a sensor may run out of the energy before its battery is recharged, causing an interruption in health monitoring service. Such service interruption may be fatal if the critical data for a severe patient is not captured in time. The second challenge is Quality of Service (QoS) guarantee for the delivery of data streams. The data streams acquired by the sensors have different priorities. For example, heart activity readings (e.g., Electrocardiography (ECG) waveforms) are often 


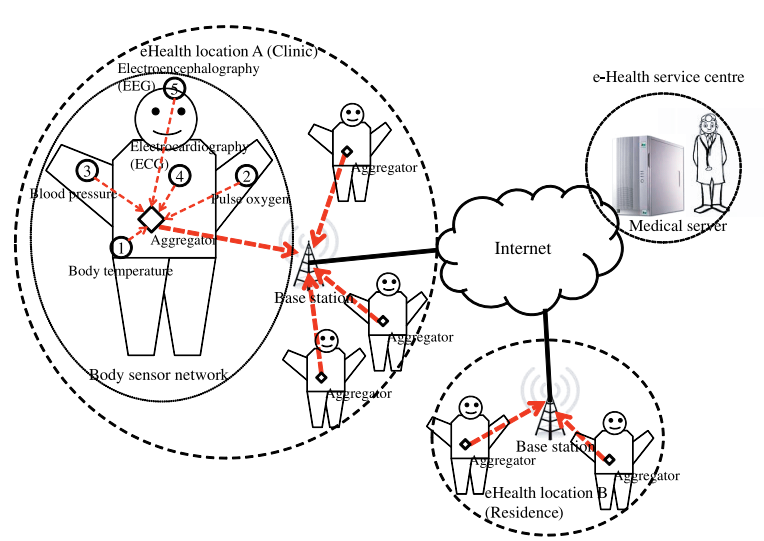

Fig. 1. A pervasive health monitoring system with body sensor networks.

considered more important than body temperature readings. In health monitoring systems, a loss or an excessive delay of the prioritized data may cause a fatal accident. However, it is quite challenging to ensure reliable data transmission in wireless communications, especially when the channel is impaired by interference or fading.

In this paper, we optimize the resource allocation in the health monitoring system to provide a sustainable and highquality service to subscribers. Our contributions in this paper are listed as follows:

1. We model the energy harvesting process at each sensor as a discrete-time Markov chain, and then theoretically analyze the relationship between the source rate and the uninterrupted lifetime of the sensor. We also formulate the steady-rate optimization problem, which minimizes the rate fluctuation with respect to the average sustainable rate, subject to the requirement of the uninterrupted service. The optimization problem is solved analytically.

2. We jointly optimize the transmit power and the transmission rate at each aggregator in an eHealth location to provide QoS guarantee to the delivery of data streams. The QoS optimization problem is converted into a convex optimization problem, which is then solved efficiently.

The remainder of this paper is organized as follows: Section 2 discusses the related work. Section 3 presents the problem statement. In Section 4, we solve the steady-rate optimization problem. In Section 5, we solve the QoS optimization problem. The simulation results are provided in Section 6, and the conclusions are drawn in Section 7.

\section{Related Work}

\subsection{Health Monitoring Systems with Body Sensor Networks}

Health monitoring systems with body sensor networks have been studied in the recent years. A heterogeneous wireless access-based remote patient monitoring system was presented in [5], in which the authors not only optimized the number of the reserved connections to minimize the service cost for the eHealth provider, but also optimized the scheduling of bio-signal data with different priority to minimize the connection cost and satisfy the delay requirements. The implementation issues for health monitoring systems were described in [15]. BSNs can also be applied to wireless monitoring of human emotions [38]. QoS support is an important issue in health monitoring systems. In [16], the authors proposed a rate control algorithm based on the $Q$-learning approach to meet medical QoS requirements in ultrasound video streaming over wireless systems. Zhou et al. proposed BodyQoS, a radio-agnostic QoS for BSNs based on a common virtual MAC abstraction [11]. A Distributed Queuing Body Area Network (DQBAN) MAC protocol was proposed in [12] to guarantee that all packets are served with their particular application-dependant QoS requirements, without endangering the battery lifetime of the body sensors in BSNs.

\subsection{Energy Harvesting in Wireless Sensor Networks}

Energy harvesting is a promising technology for many sensing applications, especially for those in which the battery replacement is impossible. A survey of the research in wireless sensor networks powered by ambient energy harvesting can be found in [17]. Adaptive power management in energy-harvesting wireless sensor networks can be found in [35], [36], and [37]. Energy-harvesting enables a new mode of operation, namely, the energy-neutral mode, at which the system uses only as much energy as available from the environment [10]. Due to the low recharging rates and the dynamics of energy harvesting process, it is a challenging task to provide services without interruption caused by exhausted battery. In [18], the authors computed the lexicographically maximum data rate for each sensor, under the constraint that no sensor will ever run out of energy. The paper in [39] outlined several systems aiming at generating electrical energy by passively tapping a variety of human body sources and activities. Energy harvesting in BSNs has been studied in [8] and [9]. In [8], the energy harvesting process is modeled by a Markov chain, based on which the probability of event loss due to energy run out is calculated. In [9], the authors developed an efficient transmission strategy for BSNs with energy harvesting capabilities, considering the trade-off between the energy consumption and packet error probability. Our work is different from the work in [8] and [9] as follows: First, we model the power consumption of a sensor and examine the relationship between the source rate and the lifetime of the sensor, which have not been studied in [8] and [9]. Second, we study the resource allocations of the health monitoring system consisting of three hops of communications, while the work in [8] and [9] only focuses on the BSN (e.g., the first-hop communications). Compared to the work in [8] and [9], our work demonstrates the following novelties: 1) we analyze the relationship between the source rate and the uninterrupted lifetime of the sensor, and optimize the source rate of each sensor to minimize the rate fluctuation under the requirement of the uninterrupted service; 2 ) we jointly optimize the transmit power and the transmission rate at each aggregator in an eHealth location to provide QoS guarantee to the delivery of data streams.

\subsection{Resource Optimizations in Wireless Networks}

Various optimization techniques have been employed in wireless networks to optimize the resource allocations [13], [14], [19], [20], [21]. A survey on network utility maximization using convex optimization can be found in [19]. In wireless cellular or ad hoc networks, a variety of power 
First-hop communications Second-hop communications Third-hop communications

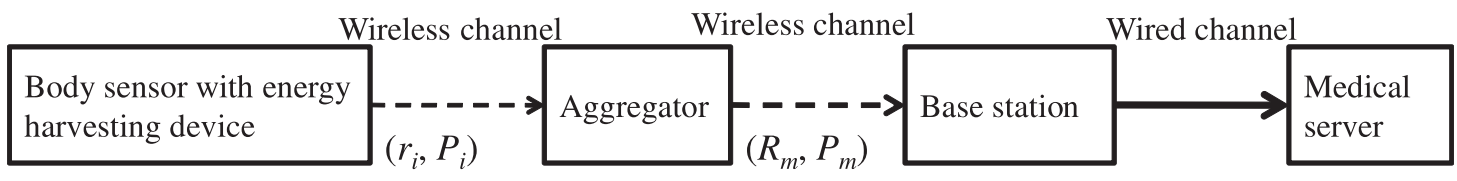

Fig. 2. Transmission path of data streams in the health monitoring system.

control problems can be formulated into Geometric Programming (GP) problems, which can be solved efficiently [20]. In [14], energy consumption is minimized by jointly optimizing the transmit power and the transmission time in Code Division Multiple Access (CDMA) wireless sensor networks. In [21], the authors proposed a game theoretic model based power regulation algorithm to reduce the bioeffects in body sensor networks.

\section{Problem Statement}

In a health monitoring system with body sensor networks, the data streams collected by the sensors are delivered to the medical server in real time. The end-to-end transmission path of data streams is illustrated in Fig. 2. The end-to-end delivery of data streams consists of three hops of communications: 1) the first-hop communications, which occur from a body sensor to the aggregator via wireless channels, 2) the second-hop communications, which occur from an aggregator to the base station via wireless channels, and 3) the third-hop communications, which occur from the base station to the medical server via wired channels.

The objective of health monitoring systems is to provide a sustainable and high-quality service to subscribers. A sustainable service requires that the system can run without interruption for a sufficiently long time such that sufficient data can be collected for a patient. The sensing activity at a sensor may be interrupted due to energy exhaustion. Such service interruption may be fatal if the critical data for a patient are missing. A high-quality service is determined by 1 ) the source rate from a sensor, and 2) the packet loss rate (PLR) and the delay of the data stream over the transmission path. The fidelity of the original signals is determined by the source rate. A higher source rate from a sensor can represent the original signals at a higher quality. The data streams collected at the sensors are delivered to the medical server for further analysis and decision making. QoS metrics, including PLR and delay, are used to measure the delivery quality. In real-time health monitoring systems, a lost packet or an excessively delayed packet may cause a fatal accident. Therefore, both sustainable power supply and QoS guarantee are important for health monitoring systems.

The sustainability and the high quality are two interdependent components. There are two trade-offs in the health monitoring system. The first trade-off is the interdependence between the sustainability and the high quality. A sensor can prolong its lifetime by generating a lower source rate, which, however, degrades the quality of the signals. The second trade-off is the interdependence between the source rate and the QoS. Higher source rates from sensors may cause more congestions and more transmission errors along the transposition path, which cause a higher PLR and a higher delay for the data streams.
In this paper, we study such a research problem: how to optimize the resource allocations in the health monitoring system to provide a sustainable and high-quality service to subscribers? Specifically, we investigate two trade-offs. The first trade-off is examined in the steady-rate optimization problem in Section 4, in which we optimize the source rate at each sensor with energy harvesting device to guarantee an uninterrupted service. The second trade-off is examined in the QoS optimization problem in Section 5, in which we jointly optimize the transmission rate and the transmission power at each aggregator to provide QoS guarantees to the data delivery. The notations used in this paper are summarized in Table 1.

\section{Steady-Rate Optimization Problem}

In this section, we study the relationship between the source rate and the lifetime of the sensor. Each body sensor has a limited battery capacity. We assume that an energy harvesting device is employed at each sensor to replenish the energy from the environment. The dynamic energy harvesting process leads to a dynamic energy replenishment at each sensor. In order to maintain an uninterrupted service, each sensor needs to adjust its source rate over time accordingly. In a health monitoring system, a steady source rate is desired. Therefore, we formulate the steady-rate optimization problem, which minimizes the rate fluctuation under the constraint of the uninterrupted service.

Each body sensor captures the physiological readings and packetizes them into packets, which are transmitted to the aggregator. A major difference between body sensor networks and other wireless sensor networks is that BSNs may contain critical readings, which should be picked out and then treated with a higher priority. Therefore, we implement the mechanism of differentiated treatment at each sensor. The differentiated treatment consists of two steps: packet classification and packet scheduling, as shown in Fig. 3. The data are acquired by sensor $i$ at a frequency $f_{i}$. Increasing the frequency $f_{i}$ of data acquisition can lead to an increased source rate. A physiological reading is packetized into a packet, and then stored in an incoming queue. At the step of packet classification, a classifier classifies the packets into one of the two classes: prioritized packets and normal packets, based on the preset thresholds at each sensor. If the physiological reading is in the normal range which indicates that the patient is in a normal condition, the corresponding packet will be classified into a normal packet. On the other hand, if the physiological reading is in the abnormal range which indicates that the patient is in an abnormal condition, the corresponding packet will be classified into a prioritized packet. The prioritized packets will be put into a priority queue, while the normal packets will be put into a normal queue. At the step of packet scheduling, a scheduler first chooses 
TABLE 1

Notations

\begin{tabular}{|c|c|}
\hline Symbol & Definition \\
\hline$r_{i}^{(t)}$ & Source rate of sensor $i$ at time slot $t$ \\
\hline$b_{i}$ & Average sustainable rate of sensor $i$ \\
\hline$P_{i}^{(t)}$ & Total power consumption at sensor $i$ at time slot $t$ \\
\hline$d_{i}$ & Distance between sensor $i$ and the aggregator \\
\hline $\mathbf{N}$ & Set of the sensors in the body sensor network \\
\hline$E_{i}^{(t)}$ & Energy of sensor $i$ at the beginning of time slot $t$ \\
\hline$\tau$ & Length of a time slot \\
\hline$\phi_{i}^{(t)}$ & Energy recharging rate of sensor $i$ at time slot $t$ \\
\hline$F_{i}^{(t)}$ & Amount of the energy not being collected during time slot $t$ due to battery overflow \\
\hline$E_{i}^{\min }$ & Minimum energy level required to be maintained at sensor $i$ \\
\hline$E_{i}^{\max }$ & Battery capacity of sensor $i$ \\
\hline$g_{i}^{a v g}$ & Long-term average recharging rate of sensor $i$ \\
\hline M & Set of the aggregators in the eHealth location \\
\hline$P_{m}^{(t)}$ & Transmission power at aggregator $m$ at time slot $t$ \\
\hline$R_{m}^{(t)}$ & Transmission rate at aggregator $m$ at time slot $t$ \\
\hline$y_{m}^{(t)}$ & $\begin{array}{l}\text { Received Bit-Energy-to-Interference-Density Ratio (BEIDR) at the base station from aggregator } m \\
\text { at time slot } t\end{array}$ \\
\hline$W$ & Spread-spectrum bandwidth in the CDMA model \\
\hline$N_{0}$ & Power spectrum density of the additive white Gaussian noise in the CDMA model \\
\hline$h_{m}^{(t)}$ & Channel gain from aggregator $m$ to the base station at time slot $t$ \\
\hline$e_{t h}$ & Threshold of Bit Error Rate (BER) \\
\hline$u_{m}^{(t)}$ & Service rate for the packets at aggregator $m$ at time slot $t$ \\
\hline$L_{m}$ & Average packet length at aggregator $m$ \\
\hline$T_{P, t h}$ & Threshold of the queueing delay for the prioritized packets at an aggregator \\
\hline$T_{t h}$ & Threshold of the queueing delay for all the packets at an aggregator \\
\hline$P_{P, t h}$ & Threshold of congestion PLR for the prioritized packets at an aggregator \\
\hline$P_{t h}$ & Threshold of congestion PLR for all the packets at an aggregator \\
\hline$\lambda_{P, m}^{(t)}$ & Average arrival rate of the prioritized packets at aggregator $m$ at time slot $t$ \\
\hline$\lambda_{m}^{(t)}$ & Average arrival rate of all packets at aggregator $m$ at time slot $t$ \\
\hline$\rho_{m}$ & Length of the queue at aggregator $m$ \\
\hline$P_{\max }$ & Maximum transmission power of the aggregator \\
\hline
\end{tabular}

the packets in the priority queue and puts them into the transmission queue. Only when the priority queue is empty, the packets in the normal queue can be scheduled for moving into the transmission queue. The transmission order of a packet is determined by the classifier and the

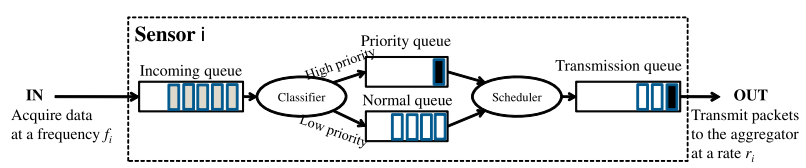

Fig. 3. Packet classification and packet scheduling at a sensor. scheduler. The packets in the transmission queue are placed based on their transmission orders. In other words, the packets to be transmitted earlier are placed in the front of the transmission queue. The sensor transmits the packets in the transmission queue to the aggregator in a First In First Out (FIFO) order.

\subsection{System Models}

\subsubsection{MAC Protocol}

IEEE 802.15.4 [30], which is specifically designed to support low power and low data rate networks, is considered as the promising standard for body sensor networks [31]. The 
IEEE 802.15.4 Personal Area Network (PAN) coordinator in a star topology can communicate with up to 255 devices [43]. IEEE 802.15.4 specifies the PHY and MAC layers of the ZigBee protocol stack [41]. The IEEE 802.15.4 MAC protocol supports two operational modes: the nonbeacon-enabled mode and the beacon-enabled mode [30]. In this paper, we adopt the beacon-enabled mode, since it has the ability of providing timely data delivery. In the beacon-enabled mode, the Beacon Interval (BI) defines the time between two consecutive beacons, and includes an active period and, optionally, an inactive period. The active period, called the superframe, is divided into 16 equally-sized time slots [30]. The lengths of the BI and the Superframe Duration (SD) are given by $B I=$ aBaseSuperframeDuration $\times 2^{B O}$ and $S D=$ aBaseSuperframeDuration $\times 2^{S O}$, respectively, where $0 \leq S O \leq B O \leq 14$, the parameter $B O$ represents the beacon order, the parameter $S O$ represents the superframe order, and the parameter aBaseSuperframeDuration denotes the minimum length of the superframe [30].

The superframe duration includes a Contention Access Period (CAP) and a Contention Free Period (CFP) [30]. The CFD consists of multiple Guaranteed Time Slots (GTSs). Each GTS may occupy one or multiple time slots in the superframe. In a BSN, each body sensor requests a dedicated GTS from the aggregator, and then transmits the data during its GTS. The GTS scheme of IEEE 802.15.4 is basically the same as the standard Time-Division Multiple Access (TDMA) scheme. Therefore, there is no interference among sensors in GTS scheme. The sensors in a BSN use beacon frames to synchronize their transmissions. Since a beacon frame is small, the overhead for synchronization is neglected in this paper.

\subsubsection{Energy Harvesting Model}

Each sensor is considered to have a rechargeable battery and an associated energy harvesting device. The harvesting device uses one of many possible underlying physical phenomena to generate energy. The recharging rate is dependent on the status of the subscriber and the ambient environment. For example, a thermal energy-harvesting device uses temperature differences between the human body and the surrounding environment to generate electricity. The status of the human body and the ambient environment is time-varying, thus the recharging rate is time-varying.

The energy harvesting process of a sensor can be studied in a discrete time manner. In each time slot, the recharging rate is assumed to be constant. Based on that, the energy harvesting process can be modeled by a discrete-time Markov chain [8], [9]. The parameters of the Markov chain, including the number of the states, the recharging rate at each state, the transition probabilities, and the duration of the time slot, should be chosen according to the characteristics of the energy harvesting hardware. There is a trade-off between the accuracy of the model and the overhead of the computations and communications. A larger number of states and a smaller duration of the time slot will improve the accuracy of the model at the expense of a higher overhead.

In this paper, we model the energy harvesting process at sensor $i$ as a discrete-time Markov chain, represented by $\left\{\mathbf{A}_{i}, \mathbf{Q}_{i}\right\}$, where $\mathbf{A}_{i}$ is the set of the states in the Markov chain, and $\mathbf{Q}_{i}$ is the transition probability matrix of the Markov chain. The recharging rate at state $m\left(m \in \mathbf{A}_{i}\right)$ is denoted by $g_{i}^{(m)}$. The states in $\mathbf{A}_{i}$ are organized in such an ascending order that $g_{i}^{(1)} \leq g_{i}^{(2)} \leq \cdots \leq g_{i}^{\left(\left|\mathbf{A}_{i}\right|\right)}$, where $\left|\mathbf{A}_{i}\right|$ is the number of the states in $\mathbf{A}_{i}$. In the transition probability matrix $\mathbf{Q}_{i}$, the element $q_{m n}$ denotes the transition probability from state $m$ to state $n$. Let $\boldsymbol{\Pi}_{i}$ denote the steady-probability vector at sensor $i$, which can be calculated from the following relationships [23]: $\boldsymbol{\Pi}_{i}^{T} \mathbf{Q}_{i}=\boldsymbol{\Pi}_{i}^{T}$, and $\boldsymbol{\Pi}_{i}^{T} \mathbf{I}=1$, where $\mathbf{I}$ is the identity vector with all elements equal to 1 . The long-term average recharging rate of sensor $i$ is then given by $g_{i}^{a v g}=\boldsymbol{\Pi}_{i}^{T} \mathbf{g}_{i}$ where $\mathbf{g}_{i}$ is the vector of the recharging rates at sensor $i$.

\subsubsection{Power Consumption Model}

In a body sensor network, the power consumption at a sensor mainly consists of two parts: sensing power consumption and transmission power consumption. The sensing power consumption at sensor $i$, denoted by $\eta_{i}$, is proportional to the source rate $r_{i}$ at sensor $i$, and it is given by $P_{s, i}=\psi_{i} r_{i}$ where $\psi_{i}$ is the energy cost for sensing at sensor $i$.

The transmission power consumption is dependent on the path loss model of the wireless channels in body sensor networks. Based on the measurement data [33], the path loss in body sensor networks can be modeled by the following equation:

$$
P L\left(d_{i}\right)=P L\left(d_{0}\right)+10 m_{p} \log _{10}\left(d_{i} / d_{0}\right)+X_{\sigma}, \quad \forall i \in \mathbf{N},
$$

where $d_{i}$ is the distance between sensor $i$ and the aggregator, $P L\left(d_{0}\right)$ is the reference path loss at distance $d_{0}\left(d_{0}=1 \mathrm{~m}\right.$ in this paper), $m_{p}$ is the path loss exponent [25], and $X_{\sigma}$ is a zero-mean Gaussian random variable. Since the Log-distance path loss model in (1) is valid in body sensor networks, we can model the transmission power at sensor $i$ by $P_{t, i}=$ $\beta_{i} r_{i}$ [24], where $\beta_{i}$ is the transmission energy consumption cost of sensor $i$, and it is given by $\beta_{i}=\theta_{i}+\zeta_{i} d_{i}^{m_{p}}$, where $\theta_{i}$ is the energy cost of transmit electronics of sensor $i$, and $\zeta_{i}$ is a coefficient term corresponding to the energy cost of transmit amplifier at sensor $i$.

The total power consumption at sensor $i$ is the sum of the sensing power and the transmission power, and it is given by

$$
\begin{aligned}
P_{i} & =P_{s, i}+P_{t, i}=\psi_{i} r_{i}+\beta_{i} r_{i} \\
& =\psi_{i} r_{i}+\left(\theta_{i}+\zeta_{i} d_{i}^{m_{p}}\right) r_{i}, \quad \forall i \in \mathbf{N} .
\end{aligned}
$$

\subsection{Analysis of the Relationship between Source Rate and Uninterrupted Lifetime of the Sensor}

A sensor consumes the energy through sensing and transmitting the data, and replenishes the energy through energy harvesting from ambient environment. Due to the time-varying energy level at each sensor, we study it in a discrete time manner. The time is evenly divided into time slots with a fixed length $\tau$. We assume that the recharging rate and the source rate at a sensor remain unchanged during a time slot.

We define the uninterrupted lifetime of a sensor as the duration from the time when the sensor starts to work until the time when the energy level of the sensor reaches 0 at the first time. Based on the energy harvesting model and the power consumption model described in Section 4.1, we 


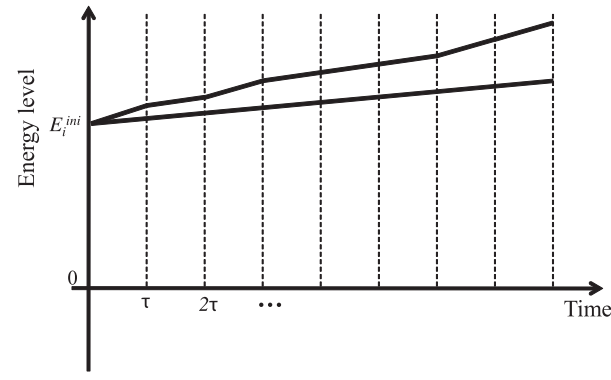

(a)

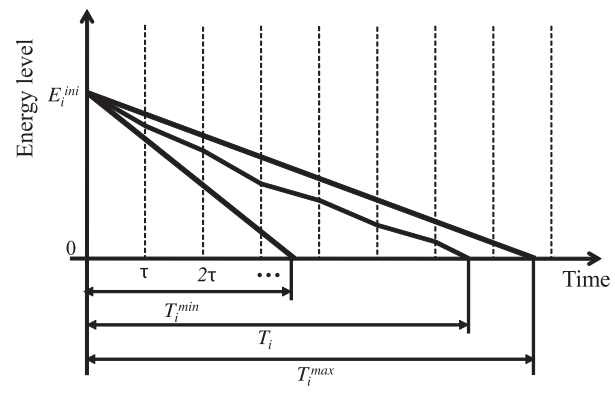

(b)

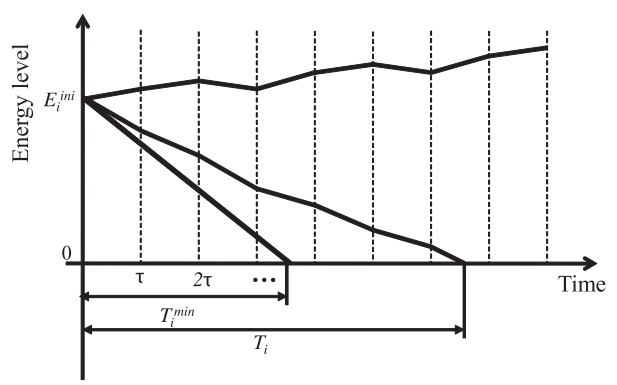

(c)

Fig. 4. Variation of the energy level at sensor $i$ with different source rate $r_{i}$ : (a) $0<r_{i} \leq \frac{g_{i}^{(1)}}{\psi_{i}+\beta_{i}}$, (b) $r_{i}>\frac{g_{i}^{\left(\left|\mathbf{A}_{i}\right|\right)}}{\psi_{i}+\beta_{i}}$, and (c) $\frac{g_{i}^{(1)}}{\psi_{i}+\beta_{i}}<r_{i} \leq \frac{g_{i}^{\left(\left|\mathbf{A}_{i}\right|\right)}}{\psi_{i}+\beta_{i}}$.

have the following theorem on the relationship between the source rate and the uninterrupted lifetime of a sensor:

Theorem 1. Given that, at sensor $i$, the initial energy is $E_{i}^{i n i}$, the source rate is $r_{i}$, the energy harvesting process is modeled by a Markov chain $\left\{\mathbf{A}_{i}, \mathbf{Q}_{i}\right\}$, and the power consumption is given by (2), then we have three statements as follows: 1) If

$$
0<r_{i} \leq \frac{g_{i}^{(1)}}{\psi_{i}+\beta_{i}},
$$

the energy level of sensor $i$ will never reach 0. 2) If $r_{i}>\frac{g_{i}^{\left(\left|\mathbf{A}_{i}\right|\right)}}{\psi_{i}+\beta_{i}}$, the energy level of sensor $i$ will definitely reach 0 at some time, and the uninterrupted lifetime of sensor $i$ is bounded by $T_{i}^{\text {min }} \leq T_{i} \leq T_{i}^{\text {max }}$, where $T_{i}^{\text {min }}=\frac{E_{i}^{\text {ini }}}{\psi_{i} r_{i}+\beta_{i} r_{i}-g_{i}^{(1)}}$ and

$$
T_{i}^{\max }=\frac{E_{i}^{i n i}}{\psi_{i} r_{i}+\beta_{i} r_{i}-g_{i}^{\left(\left|\mathbf{A}_{i}\right|\right)}} .
$$

The occurrence probability of minimum uninterrupted lifetime of sensor $i$ is given by $P_{r}\left(T_{i}=T_{i}^{\text {min }}\right)=\pi_{1} q_{11}^{\left\lfloor T_{i}^{\text {min }} / \tau\right\rfloor}$, and the occurrence probability of maximum uninterrupted lifetime of sensor $i$ is given by

$$
P_{r}\left(T_{i}=T_{i}^{\max }\right)=\pi_{\left|\mathbf{A}_{i}\right|} q_{\left|\mathbf{A}_{i} \| \mathbf{A}_{i}\right|}^{\left\lfloor T_{i}^{\max } / \tau\right\rfloor} .
$$

3) If $\frac{g_{i}^{(1)}}{\psi_{i}+\beta_{i}}<r_{i} \leq \frac{g_{i}^{\left(\left|\mathbf{A}_{i}\right|\right)}}{\psi_{i}+\beta_{i}}$, the energy level of sensor $i$ will potentially reach 0 at some time, and the uninterrupted lifetime of sensor $i$ is bounded by $T_{i} \geq T_{i}^{\text {min }}$ where

$$
T_{i}^{\text {min }}=\frac{E_{i}^{i n i}}{\psi_{i} r_{i}+\beta_{i} r_{i}-g_{i}^{(1)}} .
$$

The occurrence probability of minimum uninterrupted lifetime of sensor $i$ is given by $P_{r}\left(T_{i}=T_{i}^{\min }\right)=\pi_{1} q_{11}^{\left\lfloor T_{i}^{\min } / \tau\right\rfloor}$.
Proof. During time slot $t$, the energy increment $\epsilon_{i}$ at sensor $i$ is given by

$$
\epsilon_{i}=\tau\left(g_{i}^{(m)}-P_{i}^{(t)}\right)=\tau\left(g_{i}^{(m)}-\left(\psi_{i} r_{i}+\beta_{i} r_{i}\right)\right)
$$

where $m$ is the current Markov state. Recall that $g_{i}^{(1)} \leq$ $g_{i}^{(m)} \leq g_{i}^{\left(\left|\mathbf{A}_{i}\right|\right)}$ from the definition of $\mathbf{A}_{i}$. Therefore, we have $\tau\left(g_{i}^{(1)}-\left(\psi_{i} r_{i}+\beta_{i} r_{i}\right)\right) \leq \epsilon_{i} \leq \tau\left(g_{i}^{\left(\left|\mathbf{A}_{i}\right|\right)}-\left(\psi_{i} r_{i}+\beta_{i} r_{i}\right)\right)$.

1. If $0<r_{i} \leq \frac{g_{i}^{(1)}}{\psi_{i}+\beta_{i}}$, then $g_{i}^{(1)}-\left(\psi_{i} r_{i}+\beta_{i} r_{i}\right) \geq 0$, which means

$$
\epsilon_{i} \geq \tau\left(g_{i}^{(1)}-\left(\psi_{i} r_{i}+\beta_{i} r_{i}\right)\right) \geq 0
$$

at any time slot. In other words, the energy increment is nonnegative at any time slot, as shown in Fig. 4a. Therefore, the energy level of sensor $i$ will never reach 0 .

2. If $r_{i}>\frac{g_{i}^{\left(\left|\mathbf{A}_{i}\right|\right)}}{\psi_{i}+\beta_{i}}$, then $g_{i}^{\left(\left|\mathbf{A}_{i}\right|\right)}-\left(\psi_{i} r_{i}+\beta_{i} r_{i}\right)<0$, which means

$$
\epsilon_{i} \leq \tau\left(g_{i}^{\left(\left|\mathbf{A}_{i}\right|\right)}-\left(\psi_{i} r_{i}+\beta_{i} r_{i}\right)\right)<0
$$

at any time slot. In other words, the energy level is decreased at any time slot. Therefore, the energy level of sensor $i$ will definitely reach 0 when the initial energy is run out.

Given the initial energy $E_{i}^{i n i}$, the uninterrupted lifetime of sensor $i$ depends on the Markov state at each of the time slots from the beginning to the time when the energy is run out, as shown in Fig. $4 \mathrm{~b}$. Sensor $i$ has a minimum uninterrupted lifetime when the sequence of the Markov states is $\{1,1,1, \ldots, 1\}$ until the energy level reaches 0 at 
the first time. In this case, the energy is decreased linearly at a reduction slope $\left(\left(\psi_{i} r_{i}+\beta_{i} r_{i}\right)-g_{i}^{(1)}\right)$. Hence, the minimum uninterrupted lifetime can be found by

$$
T_{i}^{\text {min }}=\frac{E_{i}^{i n i}}{\psi_{i} r_{i}+\beta_{i} r_{i}-g_{i}^{(1)}} .
$$

Since the length of time slot is $\tau$, the number of the time slots within the minimum uninterrupted lifetime is given by $n_{\text {min }}=\left\lfloor T_{i}^{\min } / \tau\right\rfloor$ where $\lfloor x\rfloor$ is a floor function which returns the largest integer not greater than $x$. The sequence of the Markov states in this case is $\left\lfloor T_{i}^{\min } / \tau\right\rfloor$ repeated 1s. Therefore, the occurrence probability of minimum uninterrupted lifetime of sensor $i$ is given by

$$
P_{r}\left(T_{i}=T_{i}^{\text {min }}\right)=\pi_{1} q_{11}^{\left\lfloor T_{i}^{\text {min }} / \tau\right\rfloor}
$$

where $\pi_{1}$ is the probability that the sensor is initially at state 1 , and $q_{11}$ is the transition probability from state 1 to state 1 .

On the other hand, sensor $i$ has a maximum uninterrupted lifetime when the sequence of the Markov states is $\left\{\left|\mathbf{A}_{i}\right|,\left|\mathbf{A}_{i}\right|,\left|\mathbf{A}_{i}\right|, \ldots,\left|\mathbf{A}_{i}\right|\right\}$ until the energy level reaches 0 at the first time, as shown in Fig. $4 \mathrm{~b}$. In this case, the energy is decreased linearly at a reduction slope $\left(\left(\psi_{i} r_{i}+\beta_{i} r_{i}\right)-g_{i}^{\left(\left|\mathbf{A}_{i}\right|\right)}\right)$. Hence, the maximum uninterrupted lifetime can be found by $T_{i}^{\text {max }}=\frac{E_{i}^{i n i}}{\psi_{i} r_{i}+\beta_{i} r_{i}-g_{i}^{\left(\left|\mathbf{A}_{i}\right|\right)}}$. The number of the time slots within the maximum uninterrupted lifetime is given by $n_{\max }=\left\lfloor T_{i}^{\max } / \tau\right\rfloor$. Therefore, the occurrence probability of maximum uninterrupted lifetime of sensor $i$ is given by

$$
P_{r}\left(T_{i}=T_{i}^{\max }\right)=\left.\pi_{\left|\mathbf{A}_{i}\right|}\right|_{\left|\mathbf{A}_{i} \| \mathbf{A}_{i}\right|} ^{\left\lfloor T_{i}^{\max } / \tau\right\rfloor},
$$

where $\pi_{\left|\mathbf{A}_{i}\right|}$ is the probability that the sensor is initially at state $\left|\mathbf{A}_{i}\right|$, and $q_{\left|\mathbf{A}_{i} \| \mathbf{A}_{i}\right|}$ is the transition probability from state $\left|\mathbf{A}_{i}\right|$ to state $\left|\mathbf{A}_{i}\right|$.

3. If $\frac{g_{i}^{(1)}}{\psi_{i}+\beta_{i}}<r_{i} \leq \frac{g_{i}^{\left(\left|\mathbf{A}_{i}\right|\right)}}{\psi_{i}+\beta_{i}}$, then $\epsilon_{i}$ may be positive or negative or 0 at a time slot. The energy level of sensor $i$ will potentially reach 0 at some time, or will potentially never reach 0, as shown in Fig. 4c.

Sensor $i$ has a minimum uninterrupted lifetime when the sequence of the Markov states is $\{1,1,1, \ldots, 1\}$ until the energy level reaches 0 at the first time. In this case, the energy is decreased linearly at a reduction slope $\left(\left(\psi_{i} r_{i}+\beta_{i} r_{i}\right)-g_{i}^{(1)}\right)$. Hence, the minimum uninterrupted lifetime can be found by $T_{i}^{\min }=\frac{E_{i}^{i n i}}{\psi_{i} r_{i}+\beta_{i} r_{i}-g_{i}^{(1)}}$. The number of the time slots within the minimum uninterrupted lifetime is given by $n_{\min }=\left\lfloor T_{i}^{\min } / \tau\right\rfloor$. The sequence of the Markov states in this case is $\left\lfloor T_{i}^{\text {min }} / \tau\right\rfloor$ repeated $1 \mathrm{~s}$. Therefore, the occurrence probability of minimum uninterrupted lifetime of sensor $i$ is given by $P_{r}\left(T_{i}=T_{i}^{\text {min }}\right)=\pi_{1} q_{11}^{\left\lfloor T_{i}^{\min } / \tau\right\rfloor}$.

\subsection{Steady-Rate Optimization Problem}

Based on the analysis on the relationship between the source rate and the uninterrupted lifetime of a sensor in Section 4.2, we see that the uninterrupted lifetime of the sensor is dependent on the source rate. A higher source rate can increase the quality of the collected information at the price of a shorter uninterrupted lifetime.

We define the average sustainable rate $b_{i}$ of sensor $i$ as the source rate, at which sensor $i$ will consume the same energy as the harvested energy in a long run. Based on the Markov model of energy harvesting, we can find the average recharging rate $g_{i}^{\text {avg }}$ at sensor $i$. From the definition of the average sustainable rate $b_{i}$ of sensor $i$, we have $g_{i}^{a v g}=$ $\psi_{i} b_{i}+\beta_{i} b_{i}$, from which we can get

$$
b_{i}=\frac{g_{i}^{a v g}}{\psi_{i}+\beta_{i}} .
$$

The energy level may be different at the beginning of each time slot, and the recharging rate is time-varying. A sensor needs to adaptively adjust its source rate based on the remaining energy. The adjustment of the source rate at sensor $i$ can be achieved by varying the frequency of data acquisition. A steady rate from each sensor is desirable in a health monitoring system. Therefore, we set an objective to minimize the sum of the squares of the rate fluctuations of all the sensors in a BSN. The objective function at time slot $t$ is mathematically expressed by $f_{o b j}^{(t)}=\sum_{i \in \mathbf{N}}\left(r_{i}^{(t)}-b_{i}\right)^{2}$.

The steady-rate optimization problem in a body sensor network at time slot $t$ can be stated as to minimize the sum of the squares of the rate fluctuations of all the sensors, subject to the requirement of the uninterrupted service. Mathematically, the problem is formulated as follows:

$$
\begin{array}{cll}
\operatorname{minimize}_{\left(\mathbf{r}^{(t)}\right)} & \sum_{i \in \mathbf{N}}\left(r_{i}^{(t)}-b_{i}\right)^{2} & \\
\text { subject to } & P_{i}^{(t)}=\psi_{i} r_{i}^{(t)}+\beta_{i} r_{i}^{(t)}, & \forall i \in \mathbf{N}, \\
& E_{i}^{(t+1)}=E_{i}^{(t)}+\tau \phi_{i}^{(t)}-\tau P_{i}^{(t)}-F_{i}^{(t)}, & \forall i \in \mathbf{N}, \\
& E_{i}^{\min } \leq E_{i}^{(t+1)} \leq E_{i}^{\max }, & \forall i \in \mathbf{N}, \\
& r_{i}^{(t)} \geq 0, & \forall i \in \mathbf{N},
\end{array}
$$

where the optimization variable $\mathbf{r}^{(t)}$ is the vector of the source rates at time slot $t, E_{i}^{(t)}$ is the energy of sensor $i$ at the beginning of time slot $t, \phi_{i}^{(t)}$ is the energy recharging rate of sensor $i$ at time slot $t, F_{i}^{(t)}$ is the amount of the energy not being collected during time slot $t$ due to battery overflow, $E_{i}^{\text {min }}$ is the minimum energy level required to be maintained at sensor $i$, and $E_{i}^{\max }$ is the battery capacity of sensor $i$.

In the optimization problem (4), the first constraint, $P_{i}^{(t)}=\psi_{i} r_{i}^{(t)}+\beta_{i} r_{i}^{(t)}$, represents the power consumption at sensor $i$ at time slot $t$. The second constraint, $E_{i}^{(t+1)}=$ $E_{i}^{(t)}+\tau \phi_{i}^{(t)}-\tau P_{i}^{(t)}-F_{i}^{(t)}$, represents the energy level at the beginning of time slot $(t+1)$. The third constraint, 
$E_{i}^{\text {min }} \leq E_{i}^{(t+1)} \leq E_{i}^{\max }$, requires the energy level at the beginning of time slot $(t+1)$ should be no less than a minimum level $E_{i}^{\text {min }}$ and no larger than the battery capacity $E_{i}^{\max }$.

\subsection{Optimal Analytical Solution}

The optimization problem (4) can be decomposed into $|N|$ independent subproblems, where $|N|$ is the number of the sensors in the set $\mathbf{N}$. Subproblem $i$ associated with sensor $i$ is given by

$$
\begin{array}{ll}
\operatorname{minimize}_{\left(r_{i}^{(t)}\right)} & \left(r_{i}^{(t)}-b_{i}\right)^{2} \\
\text { subject to } & P_{i}^{(t)}=\psi_{i} r_{i}^{(t)}+\beta_{i} r_{i}^{(t)}, \\
& E_{i}^{(t+1)}=E_{i}^{(t)}+\tau \phi_{i}^{(t)}-\tau P_{i}^{(t)}-F_{i}^{(t)}, \\
& E_{i}^{\text {min }} \leq E_{i}^{(t+1)} \leq E_{i}^{\max }, \\
& r_{i}^{(t)} \geq 0 .
\end{array}
$$

By solving the subproblem (5) for any $i \in \mathbf{N}$, respectively, we can get the optimal solution to the problem (4). The subproblem (5) actually aims to find the source rate $r_{i}^{(t)}$ closest to the average sustainable rate $b_{i}$ based on the energy $E_{i}^{(t)}$ at the beginning of time slot $t$ and the recharging rate $\phi_{i}^{(t)}$ during time slot $t$. The optimal source rate $r_{i}^{(t)}$ for the subproblem (5) can be found by an analytical solution given as follows:

- Case 1. If $\phi_{i}^{(t)}<g_{i}^{a v g}$,

- $\quad$ Case 1.1: If $E_{i}^{(t)}-E_{i}^{\text {min }} \geq \tau\left(g_{i}^{\text {avg }}-\phi_{i}^{(t)}\right)$, the optimal source rate $r_{i}^{(t)^{*}}=b_{i}$, the objective function $f_{s u b}^{(i)}$ for the subproblem (5) is $f_{\text {sub }}^{(i)}=\left(b_{i}-b_{i}\right)^{2}=0$, and the energy at the beginning of time slot $(t+1)$ will be $E_{i}^{(t+1)}=E_{i}^{(t)}+\tau\left(\phi_{i}^{(t)}-g_{i}^{a v g}\right)$.

- Case 1.2: If $E_{i}^{(t)}-E_{i}^{\text {min }}<\tau\left(g_{i}^{\text {avg }}-\phi_{i}^{(t)}\right)$, the optimal source rate

$$
r_{i}^{(t)^{*}}=\frac{E_{i}^{(t)}-E_{i}^{\min }+\tau \phi_{i}^{(t)}}{\tau\left(\psi_{i}+\beta_{i}\right)},
$$

the objective function $f_{s u b}^{(i)}$ for the subproblem (5) is

$$
f_{\text {sub }}^{(i)}=\left(b_{i}-\frac{E_{i}^{(t)}-E_{i}^{\text {min }}+\tau \phi_{i}^{(t)}}{\tau\left(\psi_{i}+\beta_{i}\right)}\right)^{2},
$$

and the energy at the beginning of time slot $(t+1)$ will be $E_{i}^{(t+1)}=E_{i}^{\text {min }}$.

- Case 2. If $\phi_{i}^{(t)} \geq g_{i}^{a v g}$,

- $\quad$ Case 2.1: If $E_{i}^{\text {max }}-E_{i}^{(t)} \geq \tau\left(\phi_{i}^{(t)}-g_{i}^{a v g}\right)$, the optimal source rate $r_{i}^{(t)^{*}}=b_{i}$, the objective function $f_{s u b}^{(i)}$ for the subproblem (5) is $f_{s u b}^{(i)}=0$, and the energy at the beginning of time slot $(t+1)$ will be $E_{i}^{(t+1)}=E_{i}^{(t)}+\tau\left(\phi_{i}^{(t)}-g_{i}^{a v g}\right)$.

- $\quad$ Case 2.2: If $E_{i}^{\text {max }}-E_{i}^{(t)}<\tau\left(\phi_{i}^{(t)}-g_{i}^{a v g}\right)$, the optimal source rate $r_{i}^{(t)^{*}}=b_{i}$, the objective function $f_{\text {sub }}^{(i)}$ for the subproblem (5) is $f_{s u b}^{(i)}=0$, and the energy at the beginning of time slot $(t+1)$ will be
$E_{i}^{(t+1)}=E_{i}^{\max }$. In this case, the amount of wasted energy due to battery overflow is $F_{i}^{(t)}=\tau\left(\phi_{i}^{(t)}-\right.$ $\left.g_{i}^{a v g}\right)-\left(E_{i}^{\max }-E_{i}^{(t)}\right)$

Theorem 2. The proposed analytical solution is the optimal solution to the optimization problem (5).

Proof. The objective function in the optimization problem (5) is $f_{\text {sub }}^{(i)}=\left(b_{i}-r_{i}^{(t)}\right)^{2} \geq 0$. In Cases 1.1, 2.1, and 2.2 of the proposed analytical solution, the obtained objective values are all 0 . If the source rates obtained from Cases 1.1, 2.1, and 2.2 of the analytical solution satisfy the constraints of the optimization problem (5), they will be the optimal solution to the optimization problem (5).

In Case 1.1, where $\phi_{i}^{(t)}<g_{i}^{a v g}$ and $E_{i}^{(t)}-E_{i}^{\min } \geq$ $\tau\left(g_{i}^{a v g}-\phi_{i}^{(t)}\right)$, the source rate obtained from the analytical solution is $r_{i}^{(t)^{*}}=b_{i}$. The energy consumption during time slot $t$ at sensor $i$ is $E_{c o n, i}^{(t)}=\tau\left(\psi_{i}+\beta_{i}\right) b_{i}$. The energy replenishment during time slot $t$ at sensor $i$ is $E_{r e p, i}^{(t)}=\tau \phi_{i}^{(t)}$. Then, the energy increment during time slot $t$ at sensor $i$ is given by $E_{i n c, i}^{(t)}=E_{\text {rep }, i}^{(t)}-E_{c o n, i}^{(t)}$. From (3), we have $b_{i}=g_{i}^{a v g} /\left(\psi_{i}+\beta_{i}\right)$. Therefore,

$$
\begin{aligned}
E_{\text {inc }, i}^{(t)} & =E_{\text {rep }, i}^{(t)}-E_{\text {con }, i}^{(t)}=\tau \phi_{i}^{(t)}-\tau\left(\psi_{i}+\beta_{i}\right) b_{i} \\
& =\tau \phi_{i}^{(t)}-\tau\left(\psi_{i}+\beta_{i}\right)\left(g_{i}^{\text {avg }} /\left(\psi_{i}+\beta_{i}\right)\right) \\
& =\tau\left(\phi_{i}^{(t)}-g_{i}^{a v g}\right)<0 .
\end{aligned}
$$

Therefore, the amount of wasted energy due to battery overflow is zero, which means $F_{i}^{(t)}=0$. The energy at the beginning of time slot $(t+1)$ is given by

$$
\begin{aligned}
E_{i}^{(t+1)} & =E_{i}^{(t)}+\tau \phi_{i}^{(t)}-\tau\left(\psi_{i}+\beta_{i}\right) b_{i}-F_{i}^{(t)} \\
& =E_{i}^{(t)}+\tau\left(\phi_{i}^{(t)}-g_{i}^{a v g}\right)<E_{i}^{(t)} \leq E_{i}^{\max } .
\end{aligned}
$$

Since $E_{i}^{(t)}-E_{i}^{\text {min }} \geq \tau\left(g_{i}^{\text {avg }}-\phi_{i}^{(t)}\right)$, we have $E_{i}^{(t+1)}=E_{i}^{(t)}+$ $\tau\left(\phi_{i}^{(t)}-g_{i}^{a v g}\right) \geq E_{i}^{\text {min }}$. Therefore, $E_{i}^{\text {min }} \leq E_{i}^{(t+1)} \leq E_{i}^{\text {max }}$. The source rate $b_{i}$ obtained from the analytical solution satisfies the constraints of the optimization problem (5), and it achieves the minimum objective value of 0 . Therefore, it is the optimal solution to the optimization problem (5).

In Case 2.1, where $\phi_{i}^{(t)} \geq g_{i}^{a v g}$ and $E_{i}^{\max }-E_{i}^{(t)} \geq$ $\tau\left(\phi_{i}^{(t)}-g_{i}^{a v g}\right)$, the source rate obtained from the analytical solution is $r_{i}^{(t)^{*}}=b_{i}$. The energy increment during time slot $t$ at sensor $i$ is given by $E_{\text {inc }, i}^{(t)}=E_{\text {rep }, i}^{(t)}-$ $E_{c o n, i}^{(t)}=\tau \phi_{i}^{(t)}-\tau\left(\psi_{i}+\beta_{i}\right) b_{i}=\tau\left(\phi_{i}^{(t)}-g_{i}^{a v g}\right) \geq 0$. Since $E_{i}^{\max }-$ $E_{i}^{(t)} \geq \tau\left(\phi_{i}^{(t)}-g_{i}^{a v g}\right)$, the energy level won't reach the battery capacity at the end of time slot $t$. Therefore, $F_{i}^{(t)}=0$. The energy at the beginning of time slot $(t+1)$ is given by $E_{i}^{(t+1)}=E_{i}^{(t)}+\tau \phi_{i}^{(t)}-\tau\left(\psi_{i}+\beta_{i}\right) b_{i}-F_{i}^{(t)}=$ $E_{i}^{(t)}+\tau\left(\phi_{i}^{(t)}-g_{i}^{a v g}\right) \geq E_{i}^{(t)} \geq E_{i}^{\text {min }}$. From $E_{i}^{\max }-E_{i}^{(t)} \geq$ $\tau\left(\phi_{i}^{(t)}-g_{i}^{a v g}\right)$, we have $E_{i}^{(t+1)}=E_{i}^{(t)}+\tau\left(\phi_{i}^{(t)}-g_{i}^{a v g}\right) \leq E_{i}^{\text {max }}$. Therefore, $E_{i}^{\min } \leq E_{i}^{(t+1)} \leq E_{i}^{\max }$. The source rate $b_{i}$ obtained from the analytical solution satisfies the constraints of the optimization problem (5), and it 
achieves the minimum objective value of 0 . Therefore, it is the optimal solution to the optimization problem (5).

In Case 2.2, where $\phi_{i}^{(t)} \geq g_{i}^{a v g}$ and $E_{i}^{\max }-E_{i}^{(t)}<$ $\tau\left(\phi_{i}^{(t)}-g_{i}^{a v g}\right)$, the source rate obtained from the analytical solution is $r_{i}^{(t)^{*}}=b_{i}$. The energy increment during time slot $t$ at sensor $i$ is given by $E_{\text {inc, } i}^{(t)}=E_{\text {rep }, i}^{(t)}-$ $E_{\text {con }, i}^{(t)}=\tau \phi_{i}^{(t)}-\tau\left(\psi_{i}+\beta_{i}\right) b_{i}=\tau\left(\phi_{i}^{(t)}-g_{i}^{a v g}\right) \geq 0$. Since $E_{i}^{\max }-$ $E_{i}^{(t)}<\tau\left(\phi_{i}^{(t)}-g_{i}^{a v g}\right)$, the energy level will exceed the battery capacity at the end of time slot $t$, and the wasted energy due to battery overflow is given by $F_{i}^{(t)}=E_{i}^{(t)}+$ $\tau\left(\phi_{i}^{(t)}-g_{i}^{a v g}\right)-E_{i}^{\max }$. The energy at the beginning of time slot $(t+1)$ will be $E_{i}^{(t+1)}=E_{i}^{\text {max }}>E_{i}^{\text {min }}$. The source rate $b_{i}$ obtained from the analytical solution satisfies the constraints of the optimization problem (5), and it achieves the minimum objective value of 0 . Therefore, it is the optimal solution to the optimization problem (5).

In Case 1.2, where $\phi_{i}^{(t)}<g_{i}^{a v g}$ and $E_{i}^{(t)}-E_{i}^{\min }<$ $\tau\left(g_{i}^{a v g}-\phi_{i}^{(t)}\right)$, the source rate obtained from the analytical solution is $r_{i}^{(t)^{*}}=\frac{E_{i}^{(t)}-E_{i}^{\min }+\tau \phi_{i}^{(t)}}{\tau\left(\psi_{i}+\beta_{i}\right)}$. We first verify if $r_{i}^{(t)^{*}}$ is a feasible point of the optimization problem (5). The energy increment during time slot $t$ at sensor $i$ is given by

$$
\begin{aligned}
E_{\text {inc }, i}^{(t)} & =E_{\text {rep }, i}^{(t)}-E_{c o n, i}^{(t)}=\tau \phi_{i}^{(t)}-\tau\left(\psi_{i}+\beta_{i}\right) r_{i}^{(t)^{*}} \\
& =\tau \phi_{i}^{(t)}-\tau\left(\psi_{i}+\beta_{i}\right) \frac{E_{i}^{(t)}-E_{i}^{m i n}+\tau \phi_{i}^{(t)}}{\tau\left(\psi_{i}+\beta_{i}\right)} \\
& =-\left(E_{i}^{(t)}-E_{i}^{m i n}\right) \leq 0 .
\end{aligned}
$$

Hence $F_{i}^{(t)}=0$. The energy at the beginning of time slot $(t+1)$ is given by $E_{i}^{(t+1)}=E_{i}^{(t)}+E_{i n c, i}^{(t)}=E_{i}^{(t)}-$ $\left(E_{i}^{(t)}-E_{i}^{\min }\right)=E_{i}^{\min }<E_{i}^{\max }$. The source rate

$$
r_{i}^{(t)^{*}}=\frac{E_{i}^{(t)}-E_{i}^{\min }+\tau \phi_{i}^{(t)}}{\tau\left(\psi_{i}+\beta_{i}\right)}
$$

obtained from the analytical solution satisfies the constraints of the optimization problem (5), therefore it is a feasible point of the optimization problem (5). We next prove that the objective function achieves the minimum value at the feasible point

$$
r_{i}^{(t)^{*}}=\frac{E_{i}^{(t)}-E_{i}^{\min }+\tau \phi_{i}^{(t)}}{\tau\left(\psi_{i}+\beta_{i}\right)} .
$$

Since $0 \leq E_{i}^{(t)}-E_{i}^{\text {min }}<\tau\left(g_{i}^{\text {avg }}-\phi_{i}^{(t)}\right)$, we have

$$
\begin{aligned}
b_{i}-r_{i}^{(t)^{*}} & =\frac{g_{i}^{a v g}}{\psi_{i}+\beta_{i}}-\frac{E_{i}^{(t)}-E_{i}^{\text {min }}+\tau \phi_{i}^{(t)}}{\tau\left(\psi_{i}+\beta_{i}\right)} \\
& =\frac{\tau\left(g_{i}^{\text {avg }}-\phi_{i}^{(t)}\right)-\left(E_{i}^{(t)}-E_{i}^{\text {min }}\right)}{\tau\left(\psi_{i}+\beta_{i}\right)} \\
& >\frac{\tau\left(g_{i}^{\text {avg }}-\phi_{i}^{(t)}\right)-\tau\left(g_{i}^{a v g}-\phi_{i}^{(t)}\right)}{\tau\left(\psi_{i}+\beta_{i}\right)}=0 .
\end{aligned}
$$

We assume that there exists another feasible point $r_{i}^{(t)^{\prime}} \neq$ $r_{i}^{(t)^{*}}$ such that the objective value at $r_{i}^{(t)^{\prime}}$ is smaller than that at $r_{i}^{(t)^{*}}$. In other words, $\left(r_{i}^{(t)^{\prime}}-b_{i}\right)^{2}<\left(r_{i}^{(t)^{*}}-b_{i}\right)^{2}$, from which we get $r_{i}^{(t)^{*}}<r_{i}^{(t)^{\prime}}<b_{i}$ or $b_{i}<r_{i}^{(t)^{\prime}}<2 b_{i}-r_{i}^{(t)^{*}}$. We examine the two cases as follows, respectively:

1. When $r_{i}^{(t)^{*}}<r_{i}^{(t)^{\prime}}<b_{i}$, the energy increment during time slot $t$ at sensor $i$ is given by

$$
\begin{aligned}
E_{\text {inc }, i}^{(t)}= & E_{\text {rep }, i}^{(t)}-E_{c o n, i}^{(t)}=\tau \phi_{i}^{(t)}-\tau\left(\psi_{i}+\beta_{i}\right) r_{i}^{(t)^{\prime}} \\
< & \tau \phi_{i}^{(t)}-\tau\left(\psi_{i}+\beta_{i}\right) r_{i}^{(t)^{*}}=\tau \phi_{i}^{(t)} \\
& -\tau\left(\psi_{i}+\beta_{i}\right) \frac{E_{i}^{(t)}-E_{i}^{m i n}+\tau \phi_{i}^{(t)}}{\tau\left(\psi_{i}+\beta_{i}\right)} \\
= & -\left(E_{i}^{(t)}-E_{i}^{m i n}\right) .
\end{aligned}
$$

The energy at the beginning of time slot $(t+1)$ is given by $E_{i}^{(t+1)}=E_{i}^{(t)}+E_{\text {inc,i }}^{(t)}<E_{i}^{(t)}-\left(E_{i}^{(t)}-\right.$ $\left.E_{i}^{\text {min }}\right)=E_{i}^{\text {min }}$.

2. When $b_{i}<r_{i}^{(t)^{\prime}}<2 b_{i}-r_{i}^{(t)^{*}}$, the energy increment during time slot $t$ at sensor $i$ is given by

$$
\begin{aligned}
E_{\text {inc }, i}^{(t)} & =E_{\text {rep }, i}^{(t)}-E_{\text {con }, i}^{(t)}=\tau \phi_{i}^{(t)}-\tau\left(\psi_{i}+\beta_{i}\right) r_{i}^{(t)^{\prime}} \\
& <\tau \phi_{i}^{(t)}-\tau\left(\psi_{i}+\beta_{i}\right) b_{i}=\tau\left(\phi_{i}^{(t)}-g_{i}^{\text {avg }}\right) \\
& <-\left(E_{i}^{(t)}-E_{i}^{\text {min }}\right) .
\end{aligned}
$$

The energy at the beginning of time slot $(t+1)$ is given by $E_{i}^{(t+1)}=E_{i}^{(t)}+E_{\text {inc,i }}^{(t)}<E_{i}^{(t)}-\left(E_{i}^{(t)}-\right.$ $\left.E_{i}^{\min }\right)=E_{i}^{\min }$.

We have $E_{i}^{(t+1)}<E_{i}^{\text {min }}$ when $r_{i}^{(t)^{*}}<r_{i}^{(t)^{\prime}}<b_{i}$ or $b_{i}<$ $r_{i}^{(t)^{\prime}}<2 b_{i}-r_{i}^{(t)^{*}}$. Therefore, $r_{i}^{(t)^{\prime}}$ is not a feasible point of the optimization problem (5). Therefore, we conclude that

$$
r_{i}^{(t)^{*}}=\frac{E_{i}^{(t)}-E_{i}^{m i n}+\tau \phi_{i}^{(t)}}{\tau\left(\psi_{i}+\beta_{i}\right)}
$$

is the optimal point in Case 1.2 of the optimization problem (5).

In summary, the source rate obtained from the proposed analytical solution is optimal for the optimization problem (5).

Since the steady-rate optimization problem (4) can be decomposed into $|N|$ independent subproblems, each of which can be solved with the proposed analytical solution, the vector of the source rates $\left\{r_{i}^{(t)^{*}} \mid \forall i \in \mathbf{N}\right\}$ is the optimal solution to the steady-rate optimization problem (4).

In pervasive health monitoring systems, the body sensors and the aggregator are fastened on the body of the patient. Random body motions of the patient will cause dynamic changes of the distance and the direction from the sensor to the aggregator. The change of the distance leads to the change of the path loss, which causes the change of the transmission power consumption at the sensor. In addition, radio irregularity, which arises from multiple factors such as variance in RF sending power and different path losses depending on the direction of propagation, is a common phenomenon in wireless sensor networks [32]. Radio irregularity in different directions also causes the variation of the transmission power consumption at the sensor. To handle the dynamically changing status, the proposed 
solution for the steady-rate optimization problem is performed in a discrete time manner. At each time slot, sensor $i$ can obtain its transmission energy consumption cost $\beta_{i}$ by measuring the transmission power for a given data sample transmitted to the aggregator. The optimal source rate of sensor $i$ is updated at each time slot, based on the remaining energy, the current recharging rate, and the transmission energy consumption cost of sensor $i$. The proposed analytical solution is quick and efficient, which enables a quick adaptation of the optimal source rates to the dynamic changes of the status. There is a trade-off in choosing the length of the time slot. We can obtain a more accurate result with smaller time slots, at the expense of a higher overhead in computations and communications.

\section{Qos Optimization Problem}

In Section 4, we optimized the optimal source rate at each sensor to minimize the rate fluctuation under the constraint of the uninterrupted sensing. Each sensor in a BSN transmit the data stream at the optimized source rate to the aggregator, which then forward it to the medical server over the Internet. Currently two major wireless Internet access approaches are 3G cellular wireless networks and IEEE802.11 Wireless Local Area Networks (WLANs). In this paper, we work on pervasive health monitoring systems, in which the subscribers are allowed to move everywhere while being monitored. Therefore, we choose 3G cellular wireless networks as the Internet access networks. The eHealth service provider reserves a dedicated 3G frequency band for eHealth data communications. An eHealth location is a $3 \mathrm{G}$ cell area which provides eHealth service.

In health monitoring systems, a loss or an excessive delay of the prioritized data may cause a fatal accident. As shown in Fig. 2, the end-to-end transmission path of data streams consists of three hops of communications. We assume that there is no packet loss on the third-hop communications over the wired channel. Compared to the first-hop communications, the second-hop communications have a higher probability in suffering a packet loss or a long delay because: 1) an aggregator transmits a larger amount of traffic than a body sensor, which leads to a higher probability in congestion loss or congestion delay, and 2) the interferences from other users may corrupt the packets, while the interferences do not exist on first-hop communications using IEEE 802.15.4 GTS scheme. Therefore, we study the QoS optimization problem on the second-hop communications in this section.

\subsection{System Models}

\subsubsection{Queuing Model}

The aggregator aggregates the packets from all the body sensors worn by a patient. The consecutively arriving two packets at an aggregator may be sent from two different sensors. Therefore, the interarrival time between two consecutive packets at an aggregator is not constant. Instead, the interarrival time is a random variable, which can be modeled as an exponential random variable in BSN applications [40]. In order to study the QoS using queuing theory, we model the interarrival time at an aggregator as an exponential random variable. Moreover, we assume that the arrivals of the packets follow a Poisson process with a rate $\lambda_{m}^{(t)}$, the arrivals of the prioritized packets follow a Poisson process with a rate $\lambda_{P, m}^{(t)}$, and the arrivals of the normal packets follow a Poisson process with a rate $\lambda_{N, m}^{(t)}$, respectively, at aggregator $m$ during time slot $t$. Based on decomposition of Poisson process [23], we have $\lambda_{m}^{(t)}=\lambda_{P, m}^{(t)}+\lambda_{N, m}^{(t)}$. We model each aggregator as an $\mathrm{M} / \mathrm{M} / 1$ queuing system with preemptive priority service [27], in which a prioritized packet will always be scheduled for transmission once it arrives at the queue, and normal packets can be transmitted only after all of waiting prioritized packets have left the queue.

The transmission rate at aggregator $m$ at time slot $t$ is denoted by $R_{m}^{(t)}$. The service rate $u_{m}^{(t)}$ for the packets at aggregator $m$ at time slot $t$ is given by $u_{m}^{(t)}=R_{m}^{(t)} / L_{m}$ where $L_{m}$ is the average packet length at aggregator $m$. The $\mathrm{M} / \mathrm{M} / 1$ queuing system needs to satisfy the following condition in order to be stable [27]:

$$
\lambda_{N, m}^{(t)}+\lambda_{P, m}^{(t)} \leq u_{m}^{(t)}, \quad \forall m \in \mathbf{M},
$$

where $\mathbf{M}$ is the set of the aggregators in an eHealth location.

In the queuing theory, the queuing delay of a packet is defined as the duration from the time when the packet arrives in the queue to the time when the packet leaves the queue. In the M/M/1 queuing model, the average queuing delay $T_{P, m}^{(t)}$ of the prioritized packets at aggregator $m$ at time slot $t$ is given by [27]

$$
T_{P, m}^{(t)}=\frac{1 / u_{m}^{(t)}}{1-\lambda_{P, m}^{(t)} / u_{m}^{(t)}}, \quad \forall m \in \mathbf{M} .
$$

The average queuing delay $T_{m}^{(t)}$ of all packets including prioritized packets and normal packets at aggregator $m$ at time slot $t$ is given by [27]

$$
T_{m}^{(t)}=\frac{1 / u_{m}^{(t)}}{1-\lambda_{m}^{(t)} / u_{m}^{(t)}}, \quad \forall m \in \mathbf{M} .
$$

In the $M / M / 1$ queuing model, the tail probability is defined as the probability that the number of packets in the system is larger than a threshold $\rho_{m}$ [27]. Let the threshold $\rho_{m}$ represent the length of the queue at aggregator $m$. The tail probability represents the packet drop probability due to queue overflow. The tail probability $P_{P, m}^{c}$ for the prioritized packets at aggregator $m$ at time slot $t$ is given by [27]

$$
P_{P, m}^{c}=P_{r}\left(N_{P, m}>\rho_{m}\right)=\left(\lambda_{P, m}^{(t)} / u_{m}^{(t)}\right)^{\left(\rho_{m}+1\right)}, \quad \forall m \in \mathbf{M},
$$

where $N_{P, m}$ is a random variable representing the number of the prioritized packets at aggregator $m$. The tail probability $P_{m}^{c}$ for all packets at aggregator $m$ at time slot $t$ is given by [27]

$$
P_{m}^{c}=P_{r}\left(N_{m}>\rho_{m}\right)=\left(\lambda_{m}^{(t)} / u_{m}^{(t)}\right)^{\left(\rho_{m}+1\right)}, \quad \forall m \in \mathbf{M},
$$

where $N_{m}$ is a random variable representing the number of the packets at aggregator $m$. 


\subsubsection{CDMA Model}

CDMA is a spread spectrum multiple access technique, which allows multiple users to transmit the data streams to the base station simultaneously over the same physical channel. CDMA has been widely used in existing 3G cellular wireless systems.

In the CDMA model, the spread-spectrum bandwidth is denoted by $W$, the power spectrum density of the Additive White Gaussian Noise (AWGN) is denoted by $N_{0}$. The channel gain from aggregator $m$ to the base station at time slot $t$ is denoted by $h_{m}^{(t)}$, which is time-varying due to random movements of the subscribers. The received BitEnergy-to-Interference-Density Ratio (BEIDR) at the base station from aggregator $m$ at time slot $t$ is denoted by $y_{m}^{(t)}$, which is given by [14]

$$
y_{m}^{(t)}=\left(\frac{W}{R_{m}^{(t)}}\right)\left(\frac{h_{m}^{(t)} P_{m}^{(t)}}{\delta \Sigma_{j \in \mathbf{M}, j \neq m} h_{j}^{(t)} P_{j}^{(t)}+N_{0} W}\right), \quad \forall m \in \mathbf{M},
$$

where $\delta$ is the orthogonality factor representing Multiple Access Interference (MAI) from the imperfect orthogonal spreading codes.

We assume Binary Phase Shift Keying (BPSK) modulation is used in the CDMA system. The Bit Error Rate (BER) of the data transmitted from aggregator $m$ to the base station is given by

$$
e_{m}=Q\left(\sqrt{2 y_{m}^{(t)}}\right), \quad \forall m \in \mathbf{M},
$$

where $Q(x)$ is a $Q$-function [23].

If a packet is received in error, it will be dropped at the base station. We assume the bit errors occur independently in a packet. Therefore, the PLR due to transmission errors of the packets from aggregator $m$ is then given by

$$
\begin{aligned}
P_{m}^{e} & =1-\left(1-e_{m}\right)^{L_{m}} \\
& =1-\left(1-Q\left(\sqrt{2 y_{m}^{(t)}}\right)\right)^{L_{m}}, \quad \forall m \in \mathbf{M},
\end{aligned}
$$

where $L_{m}$ is the number of bits for a packet at aggregator $m$.

\subsubsection{QoS Metrics}

We examine two QoS metrics, the PLR and the delay, of the prioritized packets and all the packets at the aggregator, respectively. The PLR and the delay of the prioritized packets at the aggregator indicate the delivery quality of the critical data, while the PLR and the delay of all packets at the aggregator indicate the overall transmission performance.

The PLR consists of the congestion PLR due to queue overflow and the transmission PLR due to transmission errors. The PLR of the prioritized packets at aggregator $m$, denoted by $P_{P, m}^{P L R}$, is given by

$$
\begin{aligned}
& P_{P, m}^{P L R}=1-\left(1-P_{P, m}^{c}\right)\left(1-P_{m}^{e}\right) \\
&=1-\left(1-\left(\frac{\lambda_{P, m}^{(t)}}{u_{m}^{(t)}}\right)^{\left(\rho_{m}+1\right)}\right)\left(1-Q\left(\sqrt{2 y_{m}^{(t)}}\right)\right)^{L_{m}}, \\
& \forall m \in \mathbf{M} .
\end{aligned}
$$

The PLR of all packets at aggregator $m$, denoted by $P_{m}^{P L R}$, is given by

$$
\begin{aligned}
P_{m}^{P L R}= & 1-\left(1-P_{m}^{c}\right)\left(1-P_{m}^{e}\right) \\
=1 & \left(1-\left(\frac{\lambda_{m}^{(t)}}{u_{m}^{(t)}}\right)^{\left(\rho_{m}+1\right)}\right)\left(1-Q\left(\sqrt{2 y_{m}^{(t)}}\right)\right)^{L_{m}}, \\
& \forall m \in \mathbf{M} .
\end{aligned}
$$

The delay of a packet consists of the queuing delay and the propagation delay. The propagation delay is small compared to the queuing delay, thus it is negligible. The delay of a packet is then equal to the queuing delay. The average queuing delay of the prioritized packets at aggregator $m$ is given by (7), and the average queuing delay of all packets at aggregator $m$ is given by (8).

\subsection{QoS Optimization Problem}

We optimize the resource allocation in an eHealth location to provide QoS guarantee to the delivery of the data streams. The QoS optimization problem can be stated as: to minimize the sum of the transmission powers of all the aggregators, subject to the power constraints, the requirements of the congestion PLR, the transmission BER, and the delays of the prioritized packets and all the packets, respectively. Mathematically, the problem is formulated as follows:

$$
\begin{aligned}
& \operatorname{minimize}_{\left(\mathbf{R}^{(t)}, \mathbf{P}^{(t)}\right)} \quad \sum_{m \in \mathbf{M}} P_{m}^{(t)} \\
& \text { subject to } \\
& Q\left(\sqrt{2 y_{m}^{(t)}}\right) \leq e_{t h}, \\
& y_{m}^{(t)}=\left(\frac{W}{R_{m}^{(t)}}\right)\left(\frac{h_{m}^{(t)} P_{m}^{(t)}}{\delta \Sigma_{j \in \mathbf{M}, j \neq m} h_{j}^{(t)} P_{j}^{(t)}+N_{0} W}\right), \forall m \in \mathbf{M} \\
& u_{m}^{(t)}=R_{m}^{(t)} / L_{m}, \\
& \forall m \in \mathbf{M} \text {, } \\
& \frac{1 / u_{m}^{(t)}}{1-\lambda_{P, m}^{(t)} / u_{m}^{(t)}} \leq T_{P, t h}, \\
& \forall m \in \mathbf{M} \\
& \frac{1 / u_{m}^{(t)}}{1-\lambda_{m}^{(t)} / u_{m}^{(t)}} \leq T_{t h}, \\
& \forall m \in \mathbf{M} \\
& \left(\lambda_{P, m}^{(t)} / u_{m}^{(t)}\right)^{\left(\rho_{m}+1\right)} \leq P_{P, t h}, \\
& \forall m \in \mathbf{M} \\
& \left(\lambda_{m}^{(t)} / u_{m}^{(t)}\right)^{\left(\rho_{m}+1\right)} \leq P_{t h}, \\
& \lambda_{N, m}^{(t)}+\lambda_{P, m}^{(t)} \leq u_{m}^{(t)}, \\
& \forall m \in \mathbf{M} \\
& 0 \leq P_{m}^{(t)} \leq P_{\max }, \\
& R_{m}^{(t)}>0 \text {, } \\
& \forall m \in \mathbf{M} \text {, } \\
& \forall m \in \mathbf{M} \text {, } \\
& \forall m \in \mathbf{M}
\end{aligned}
$$

where $\mathbf{R}^{(t)}$ is the vector of the transmission rates at time slot $t, \mathbf{P}^{(t)}$ is the vector of the transmission powers at time slot $t, P_{m}^{(t)}$ is the transmission power at aggregator $m$ at time slot $t, R_{m}^{(t)}$ is the transmission rate at aggregator $m$ at time slot $t, e_{t h}$ is the threshold of BER, $u_{m}^{(t)}$ is the service rate for the packets at aggregator $m$ at time slot $t, T_{P, t h}$ is the threshold of the queuing delay for the prioritized packets at an aggregator, $T_{t h}$ is the threshold of the queuing delay for all packets at an aggregator, $P_{P, t h}$ is the threshold of congestion PLR for the prioritized packets at an aggregator, $P_{t h}$ is the threshold of congestion PLR for all packets at an aggregator, and $P_{\max }$ is the maximum transmission power at an aggregator. 
$Q$ function is a monotonically decreasing function. Therefore, we can convert the constraints,

$$
\begin{aligned}
Q\left(\sqrt{2 y_{m}^{(t)}}\right) & \leq e_{t h} \text { and } y_{m}^{(t)} \\
& =\left(\frac{W}{R_{m}^{(t)}}\right)\left(\frac{h_{m}^{(t)} P_{m}^{(t)}}{\delta \Sigma_{j \in \mathrm{M}, j \neq m} h_{j}^{(t)} P_{j}^{(t)}+N_{0} W}\right)
\end{aligned}
$$

in the optimization problem (16), to an equivalent form,

$$
\left(\frac{W}{R_{m}^{(t)}}\right)\left(\frac{h_{m}^{(t)} P_{m}^{(t)}}{\delta \Sigma_{j \in \mathbf{M}, j \neq m} h_{j}^{(t)} P_{j}^{(t)}+N_{0} W}\right) \geq \frac{\left(Q^{-1}\left(e_{t h}\right)\right)^{2}}{2},
$$

where $Q^{-1}(x)$ is the inverse $Q$-function [23]. After the conversion, the optimization problem (16) is changed to the following equivalent form:

$$
\begin{aligned}
& \operatorname{minimize}_{\left(\mathbf{R}^{(t)}, \mathbf{P}^{(t)}\right)} \quad \sum_{m \in \mathbf{M}} P_{m}^{(t)} \\
& \text { subject to } \quad\left(\frac{W}{R_{m}^{(t)}}\right)\left(\frac{h_{m}^{(t)} P_{m}^{(t)}}{\delta \Sigma_{j \in \mathbf{M}, j \neq m} h_{j}^{(t)} P_{j}^{(t)}+N_{0} W}\right) \\
& \geq \frac{\left(Q^{-1}\left(e_{t h}\right)\right)^{2}}{2}, \quad \forall m \in \mathbf{M}, \\
& u_{m}^{(t)}=\frac{R_{m}^{(t)}}{L_{m}}, \quad \forall m \in \mathbf{M}, \\
& \frac{1 / u_{m}^{(t)}}{1-\lambda_{P, m}^{(t)} / u_{m}^{(t)}} \leq T_{P, t h}, \quad \forall m \in \mathbf{M}, \\
& \frac{1 / u_{m}^{(t)}}{1-\lambda_{m}^{(t)} / u_{m}^{(t)}} \leq T_{t h}, \quad \forall m \in \mathbf{M}, \\
& \left(\lambda_{P, m}^{(t)} / u_{m}^{(t)}\right)^{\left(\rho_{m}+1\right)} \leq P_{P, t h}, \quad \forall m \in \mathbf{M}, \\
& \left(\lambda_{m}^{(t)} / u_{m}^{(t)}\right)^{\left(\rho_{m}+1\right)} \leq P_{t h}, \quad \forall m \in \mathbf{M}, \\
& \lambda_{m}^{(t)} \leq u_{m}^{(t)}, \quad \forall m \in \mathbf{M}, \\
& 0 \leq P_{m}^{(t)} \leq P_{\max }, \quad \forall m \in \mathbf{M}, \\
& R_{m}^{(t)}>0, \quad \forall m \in \mathbf{M} \text {. }
\end{aligned}
$$

Let $\gamma_{t h}=\frac{\left(Q^{-1}\left(e_{t h}\right)\right)^{2}}{2}$, representing the threshold of the received BEIDR. If the received BEIDR at the base station is larger than $\gamma_{t h}$, the BER of the received signal will be less than $e_{t h}$.

In the optimization problem (17), the objective is to minimize the sum of the transmission powers of all the aggregators. The first constraint,

$$
\left(\frac{W}{R_{m}^{(t)}}\right)\left(\frac{h_{m}^{(t)} P_{m}^{(t)}}{\delta \Sigma_{j \in \mathbf{M}, j \neq m} h_{j}^{(t)} P_{j}^{(t)}+N_{0} W}\right) \geq \frac{\left(Q^{-1}\left(e_{t h}\right)\right)^{2}}{2},
$$

requires the received BEIDR at the base station from aggregator $m$ to be no less than the threshold $\gamma_{t h}$. The second constraint, $u_{m}^{(t)}=R_{m}^{(t)} / L_{m}$, represents the service rate for the packets at aggregator $m$ at time slot $t$. The third constraint, $\frac{1 / u_{m}^{(t)}}{1-\lambda_{P}^{(t)} / u_{m}^{(t)}} \leq T_{P, t h}$, requires that the prioritized packets at aggregator $m$ at time slot $t$ have an average queuing delay no larger than a threshold $T_{P, t h}$. The fourth constraint, $\frac{1 / u_{m}^{(t)}}{1-\lambda_{m}^{(t)} / u_{m}^{(t)}} \leq T_{t h}$, requires that all packets at aggregator $m$ at time slot $t$ have an average queuing delay no larger than a threshold $T_{t h}$. The fifth constraint, $\left(\lambda_{P, m}^{(t)} / u_{m}^{(t)}\right)^{\left(\rho_{m}+1\right)} \leq P_{P, t h}$, requires that the prioritized packets at aggregator $m$ at time slot $t$ have a congestion PLR no larger than a threshold $P_{P, t h}$.
The sixth constraint, $\left(\lambda_{m}^{(t)} / u_{m}^{(t)}\right)^{\left(\rho_{m}+1\right)} \leq P_{t h}$, requires that all the packets at aggregator $m$ at time slot $t$ have a congestion PLR no larger than a threshold $P_{t h}$. The seventh constraint, $\lambda_{m}^{(t)} \leq u_{m}^{(t)}$, requires that the service rate at aggregator $m$ at time slot $t$ should be no less than the arrival rate of all packets in order to maintain a stable $\mathrm{M} / \mathrm{M} / 1$ queuing system.

\subsection{Optimal Numerical Solution}

We convert the constraint,

$$
\left(\frac{W}{R_{m}^{(t)}}\right)\left(\frac{h_{m}^{(t)} P_{m}^{(t)}}{\delta \Sigma_{j \in \mathbf{M}, j \neq m} h_{j}^{(t)} P_{j}^{(t)}+N_{0} W}\right) \geq \frac{\left(Q^{-1}\left(e_{t h}\right)\right)^{2}}{2}
$$

in the optimization problem (17), to an equivalent form

$$
\frac{\delta \gamma_{t h} R_{m}^{(t)}}{h_{m}^{(t)} P_{m}^{(t)} W} \Sigma_{j \in \mathbf{M}, j \neq m} h_{j}^{(t)} P_{j}^{(t)}+\frac{\gamma_{t h} N_{0}}{h_{m}^{(t)}}\left(\frac{R_{m}^{(t)}}{P_{m}^{(t)}}\right) \leq 1 .
$$

The constraint, $\frac{1 / u_{m}^{(t)}}{1-\lambda_{P, m}^{(t)} / u_{m}^{(t)}} \leq T_{P, t h}$, is equivalent to

$$
R_{m}^{(t)} \geq \frac{L_{m}+T_{P, t h} \lambda_{P, m}^{(t)} L_{m}}{T_{P, t h}}
$$

The constraint, $\frac{1 / u_{m}^{(t)}}{1-\lambda_{m}^{(t)} / u_{m}^{(t)}} \leq T_{t h}$, is equivalent to

$$
R_{m}^{(t)} \geq \frac{L_{m}+T_{t h} \lambda_{m}^{(t)} L_{m}}{T_{t h}}
$$

The constraint, $\left(\lambda_{P, m}^{(t)} / u_{m}^{(t)}\right)^{\left(\rho_{m}+1\right)} \leq P_{P, t h}$, is equivalent to $R_{m}^{(t)} \geq \frac{\lambda_{P, m} L_{m}}{P_{P, t h}^{1 /(m+1)}}$. The constraint,

$$
\left(\lambda_{m}^{(t)} / u_{m}^{(t)}\right)^{\left(\rho_{m}+1\right)} \leq P_{t h},
$$

is equivalent to $R_{m}^{(t)} \geq \frac{\lambda_{m}^{(t)} L_{m}}{P_{t h}^{\left(/ /\left(\rho_{m}+1\right)\right.}}$. The constraint, $\lambda_{m}^{(t)} \leq u_{m}^{(t)}$, is equivalent to

$$
R_{m}^{(t)} \geq \lambda_{m}^{(t)} L_{m}
$$

Therefore, the optimization problem (17) is then converted to the following equivalent form:

$$
\begin{array}{cll}
\operatorname{minimize}_{\left(\mathbf{R}^{(t)} \mathbf{P}^{(t)}\right)} & \sum_{m \in \mathbf{M}} P_{m}^{(t)} & \\
\text { subject to } & \frac{\delta \gamma_{t h} R_{m}^{(t)}}{h_{m}^{(t)} P_{m}^{(t)} W} \sum_{j \in \mathbf{M}, j \neq m} h_{j}^{(t)} P_{j}^{(t)} & \\
& +\frac{\gamma t h N_{0}}{h_{m}^{(t)}}\left(\frac{R_{m}^{(t)}}{P_{m}^{(t)}}\right) \leq 1, & \\
& 0 \leq P_{m}^{(t)} \leq P_{\max }, & \forall m \in \mathbf{M}, \\
& R_{m}^{(t)} \geq R_{m}^{L B}, & \forall m \in \mathbf{M},
\end{array}
$$

where

$$
\begin{gathered}
R_{m}^{L B}=\max \left\{\frac{L_{m}+T_{P, t h} \lambda_{P, m}^{(t)} L_{m}}{T_{P, t h}}, \frac{L_{m}+T_{t h} \lambda_{m}^{(t)} L_{m}}{T_{t h}},\right. \\
\left.\frac{\lambda_{P, m}^{(t)} L_{m}}{P_{P, t h}^{1 /\left(\rho_{m}+1\right)}}, \frac{\lambda_{m}^{(t)} L_{m}}{P_{t h}^{1 /\left(\rho_{m}+1\right)}}, \lambda_{m}^{(t)} L_{m}\right\} .
\end{gathered}
$$

Theorem 3. The optimization problem (18) is a geometric programming that can be transformed into a convex optimization problem.

Proof. A geometric programming problem is an optimization problem of the form as follows [28]. 


$$
\begin{array}{ll}
\operatorname{minimize}_{(\mathbf{x})} & f_{0}(\mathbf{x}) \\
\text { subject to } & f_{i}(\mathbf{x}) \leq 1, \quad i=1,2, \ldots, m, \\
& g_{l}(\mathbf{x})=1, \quad l=1,2, \ldots, p,
\end{array}
$$

where $f_{i}(\mathbf{x}), i=0,1,2, \ldots, m$, are posynomials, given by

$$
f_{i}(\mathbf{x})=\sum_{k=1}^{K_{i}} d_{i k} x_{1}^{a_{i k}^{(1)}} x_{2}^{a_{i k}^{(2)}} \ldots x_{n}^{a_{i k}^{(n)}},
$$

where $d_{i k}>0$ and $a_{i k}^{(j)} \in \mathbf{R}$ for $j=1,2, \ldots, n$, and $g_{l}(\mathbf{x})$, $l=1,2, \ldots, p$, are monomials, given by

$$
g_{l}(\mathbf{x})=d_{l} x_{1}^{a_{l}^{(1)}} x_{2}^{a_{l}^{(2)}} \ldots x_{n}^{a_{l}^{(n)}}
$$

where $d_{l}>0$ and $a_{l}^{(j)} \in \mathbf{R}$ for $j=1,2, \ldots, n$.

In the optimization problem (18), the objective function is a posynomial, and the left side of the first constraint

$$
\frac{\delta \gamma_{t h} R_{m}^{(t)}}{h_{m}^{(t)} P_{m}^{(t)} W} \Sigma_{j \in \mathbf{M}, j \neq m} h_{j}^{(t)} P_{j}^{(t)}+\frac{\gamma_{t h} N_{0}}{h_{m}^{(t)}}\left(\frac{R_{m}^{(t)}}{P_{m}^{(t)}}\right)
$$

is also a posynomial. Therefore, the optimization problem (18) is a geometric programming problem.

The geometric programming problem in (18) is not a convex optimization problem, because the inequality constraint function,

$$
\frac{\delta \gamma_{t h} R_{m}^{(t)}}{h_{m}^{(t)} P_{m}^{(t)} W} \Sigma_{j \in \mathbf{M}, j \neq m} h_{j}^{(t)} P_{j}^{(t)}+\frac{\gamma_{t h} N_{0}}{h_{m}^{(t)}}\left(\frac{R_{m}^{(t)}}{P_{m}^{(t)}}\right),
$$

is not a convex function. However, with a logarithmic change of the variables, the geometric programming problem in (18) can be converted into a convex optimization problem. Let $z_{m}^{(t)}=\ln \left(R_{m}^{(t)}\right)$ and $v_{m}^{(t)}=\ln \left(P_{m}^{(t)}\right)$. Taking the natural logarithms of both the objective function and the constraints, the optimization problem (18) is converted into the following problem:

$$
\begin{aligned}
& \operatorname{minimize}_{\left(\mathbf{z}^{(t)}, \mathbf{v}^{(t)}\right)} \ln \sum_{m \in \mathbf{M}} \exp \left(v_{m}^{(t)}\right) \\
& \text { subject to } \quad \ln \left[\Sigma _ { j \in \mathbf { M } , j \neq m } \operatorname { e x p } \left(z_{m}^{(t)}\right.\right. \\
& \left.+v_{j}^{(t)}-v_{m}^{(t)}+\ln \left(\frac{\delta \gamma_{t h} h_{j}^{(t)}}{h_{m}^{(t)} W}\right)\right) \\
& +\exp \left(z_{m}^{(t)}-v_{m}^{(t)}\right. \\
& \left.\left.+\ln \left(\frac{\gamma_{t h} N_{0}}{h_{m}^{(t)}}\right)\right)\right] \leq 0, \\
& v_{m}^{(t)} \leq \ln \left(P_{\max }\right) \text {, } \\
& z_{m}^{(t)} \geq \ln \left(R_{m}^{L B}\right)
\end{aligned}
$$

Since a log-sum-exponential function

$$
f(\mathbf{x})=\log \left(\sum_{i=1}^{n} \exp \left(x_{i}\right)\right)
$$

is a convex function [29], the objective function, $\ln \sum_{m \in \mathbf{M}} \exp \left(v_{m}^{(t)}\right)$, and the inequality constraint function,

$$
\begin{aligned}
& \ln \left[\Sigma_{j \in \mathbf{M}, j \neq m} \exp \left(z_{m}^{(t)}+v_{j}^{(t)}-v_{m}^{(t)}+\ln \frac{\delta \gamma_{t h} h_{j}^{(t)}}{h_{m}^{(t)} W}\right)\right. \\
& \left.+\exp \left(z_{m}^{(t)}-v_{m}^{(t)}+\ln \frac{\gamma_{t h} N_{0}}{h_{m}^{(t)}}\right)\right],
\end{aligned}
$$

in the optimization problem (20), are both convex functions. Therefore, the optimization problem (20) is a convex optimization problem.

Efficient solution methods for convex optimization problems are well developed. We can use the primal-dual interior-point methods [29] to solve the convex optimization problem (20). The globally optimal solution to the original optimization problem (16) will be obtained by $P_{m}^{(t) *}=\exp \left(v_{m}^{(t) *}\right)$ and $R_{m}^{(t) *}=\exp \left(z_{m}^{(t) *}\right), \forall m \in \mathbf{M}$, where $v_{m}^{(t) *}$ and $z_{m}^{(t) *}$ are the optimal solution to the convex optimization problem (20).

In the protocol implementation, the base station performs the QoS optimization at each time slot. In the initialization stage, the parameters $\left(W, \delta, N_{0}\right)$ are obtained by the base station, the parameters $\left(e_{t h}, T_{P, t h}, T_{t h}, P_{P, t h}\right.$, $\left.P_{t h}, P_{\max }\right)$ are set by the administrator, and the parameters $\left(L_{m}, \rho_{m}\right)$ for aggregator $m$ are provided by aggregator $m$. At the beginning of time slot $t$, aggregator $m$ updates the parameters $\left(\lambda_{P, m}^{(t)}, \lambda_{m}^{(t)}, h_{m}^{(t)}\right)$, and sends them to the base station. The base station computes the optimal transmission rate $R_{m}^{(t) *}$ and the optimal transmission power $P_{m}^{(t) *}$ for aggregator $m(\forall m \in \mathbf{M})$ by solving the optimization problem (16), and then feeds them back to aggregator $m$, respectively. Aggregator $m$ will transmit the data stream to the base station at the optimal transmission rate $R_{m}^{(t) *}$ with the optimal transmission power $P_{m}^{(t) *}$ during time slot $t$.

\section{Simulations}

\subsection{Simulation Setting}

We perform simulations to evaluate the proposed methods. The typical body sensors in health monitoring applications include:

1. body temperature sensor,

2. pulse oxygen sensor,

3. blood pressure sensor,

4. ECG sensor, and

5. electroencephalography (EEG) sensor [34].

Therefore, we deploy the five sensors in a BSN. The five sensors and the aggregator are fastened on the front of the body. The aggregator is placed on the waist of the body. The BSN adopts IEEE 802.15.4 protocol and operates in the $2.4 \mathrm{GHz}$ frequency band with a data rate of $250 \mathrm{Kbps}$. In the MAC configuration, the values of $S O$ and $B O$ are both set to 3, each GTS occupies one time slot in the superframe. The distance from a sensor to the aggregator is uniformly distributed between 0.3 and $0.7 \mathrm{~m}$ [33]. In the power consumption model for sensor $i$, we set $\psi_{i}=2 \times 10^{-8} \mathrm{~J} / \mathrm{b}$, $\theta_{i}=4 \times 10^{-8} \mathrm{~J} / \mathrm{b}, \zeta_{i}=1.3 \times 10^{-8} \mathrm{~J} / \mathrm{b} / \mathrm{m}^{2.4}$. The path loss exponent is set to $m_{p}=2.4$ based on the measurement results in [33]. The initial energy of each sensor is set to $0.1 \mathrm{~J}$. Each sensor has a battery capacity $0.11 \mathrm{~J}$. The minimum energy required for each sensor is $0.01 \mathrm{~J}$. In body sensor networks, typical energy harvesting devices are vibration-based energy harvesters, which generate the power using human body motions during activities of daily living [42]. Therefore, we equip each body sensor with a vibration-based energy harvester. The energy harvesting process at a sensor is modeled by a two-state Markov chain 


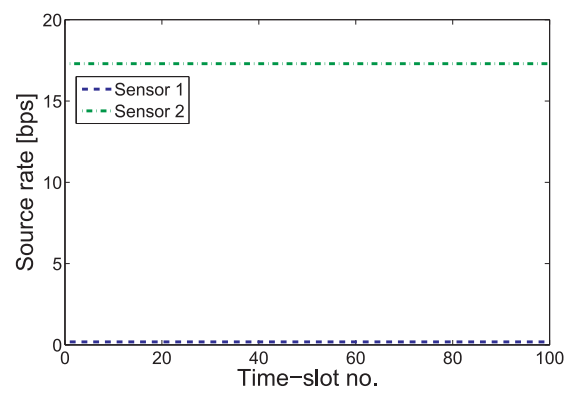

(a)

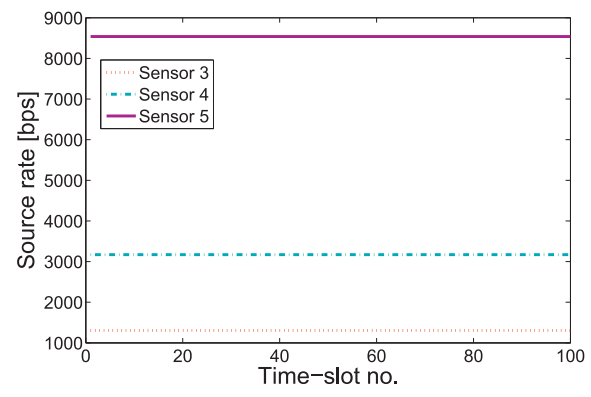

(b)

Fig. 5. Variations of the source rates during 100 time slots: (a) at sensors 1-2 and (b) at sensors 3-5.

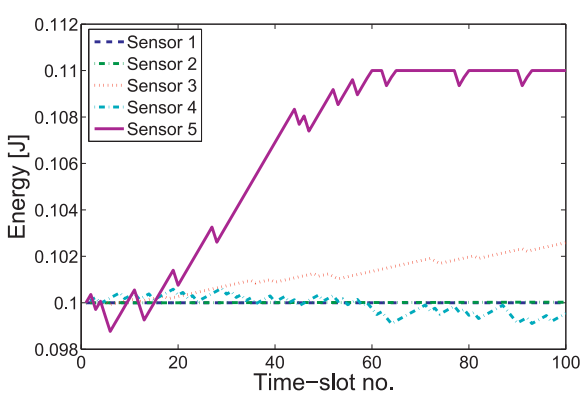

(a)

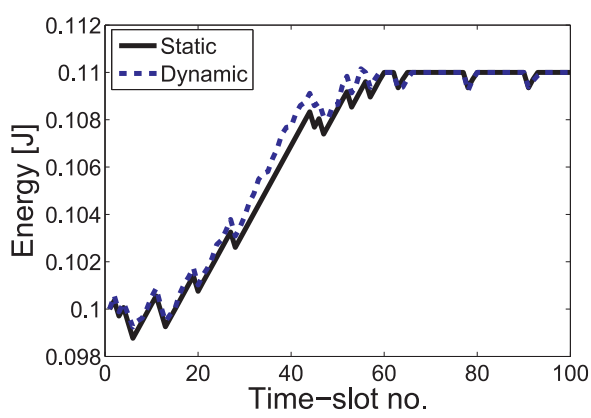

(b)

Fig. 6. Energy variations during 100 time slots: (a) at sensors 1-5 in a static state and (b) at sensor 5 in a static state and a dynamic state, respectively.

with state 1 and state 2 [8]. The transition probability from state 1 to state 2 is uniformly distributed between 0.6 and 0.8 , and the transition probability from state 2 to state 1 is uniformly distributed between 0.2 and 0.4 . A vibrationbased energy harvesters typically stays at a state for an interval in the range of 1-10 s. Therefore, we set the length of the time slot to $5 \mathrm{~s}$. During a time slot, we assume that the energy recharging rate, the distance and the direction from a sensor to the aggregator, and the distance from an aggregator to the base station remain unchanged. The aggregator aggregates the data streams from the body sensors. In an eHealth location, the number of the subscribers is set to 10 if not specified particularly, the distance from a subscriber to the base station is uniformly distributed between 50 and $200 \mathrm{~m}$. The channel gain from aggregator $m$ to the base station is given by $h_{m}=200 / d_{m}^{4}$, where $d_{m}$ is the distance from aggregator $m$ to the base station. In the CDMA model, we set $W=500 \mathrm{KHz}, \delta=0.1$, and $N_{0}=10^{-13} \mathrm{~W} / \mathrm{Hz}$. The average packet length is 200 bits for all aggregators. The length of the queue at the aggregator is set to 10 packets. In the setting of QoS thresholds, we set the threshold of transmission BER to $10^{-5}$, the threshold of the congestion PLR for the prioritized packets at an aggregator to 0.01, the threshold of the congestion PLR for all the packets at an aggregator to 0.05, the threshold of the queuing delay for the prioritized packets at an aggregator to $0.1 \mathrm{~s}$, and the threshold of the queuing delay for all the packets at an aggregator to $0.3 \mathrm{~s}$. The maximum transmission power is set to $1.0 \mathrm{~W}$ for all aggregators.

\subsection{Simulation Results}

The variations of the source rates of five body sensors in a BSN during 100 time slots is shown in Fig. 5. Each sensor minimizes the rate fluctuation with respective to the average sustainable rate, subject to the requirement of uninterrupted service. As shown in Fig. 5, each sensor maintains a constant source rate, equal to the average sustainable rate, during the 100 time slots, which indicates a steady data transmission. The source rates are heterogeneous among sensors. Sensor 1 (body temperature sensor) and sensor 2 (pulse oxygen sensor) have a much smaller source rate, shown in Fig. 5a, compared to sensor 3 (blood pressure sensor), sensor 4 (ECG sensor), and sensor 5 (EEG sensor), shown in Fig. $5 b$.

The energy level of each sensor varies over time, as shown in Fig. 6a, because the energy harvesting is a random process. When the harvested energy is larger than the consumed energy, the energy level of the sensor is increased. When the harvested energy is smaller than the consumed energy, it is decreased. The variation of the energy level is limited to the range between the battery capacity $0.11 \mathrm{~J}$ and the minimum energy $0.01 \mathrm{~J}$. During time slots 60-62, 65-77, 80-90, and 93-100, the battery of sensor 5 is overflowed, which causes the wasted energy. Fig. 6a shows the energy variations at the five sensors when the body is in a static state, in which the distance and the direction from a sensor to the aggregator keep unchanged. However, patients may have random body motions, such as body stretches, which cause dynamic changes of the distance and the direction from the sensor to the aggregator. The variation of the distance causes the 
TABLE 2

Relationship between the Source Rate and the Lifetime of Sensor 3

\begin{tabular}{|l|l|l|l|l|l|l|l|l|}
\hline Source rate [bps] & 847 & 969 & 1090 & 1211 & 1332 & 1453 & 1574 & 1695 \\
\hline Minimum uninterrupted lifetime [s] & $\infty$ & $\infty$ & 13228 & 6614 & 4409 & 3307 & 2646 & 2205 \\
\hline Maximum uninterrupted lifetime [s] & $\infty$ & $\infty$ & $\infty$ & $\infty$ & $\infty$ & $\infty$ & 13228 & 6614 \\
\hline
\end{tabular}

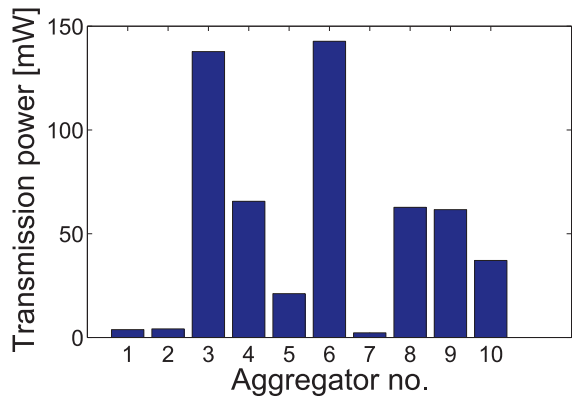

(a)

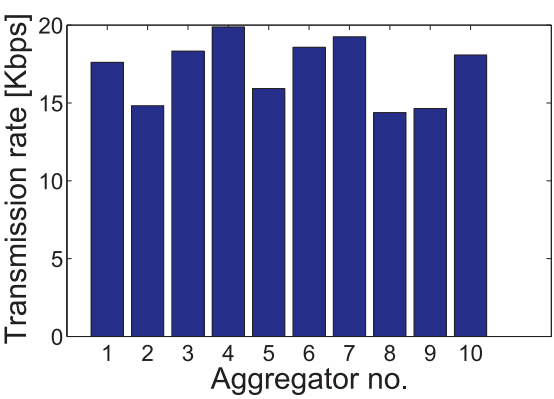

(b)

Fig. 7. Optimal results obtained from the optimized scheme in an eHealth location with 10 subscribers: (a) transmission powers and (b) transmission rates.

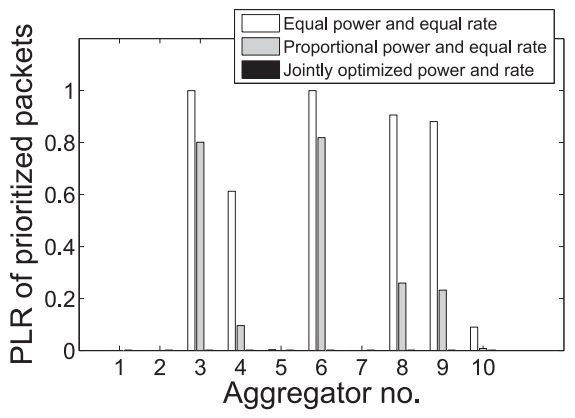

(a)

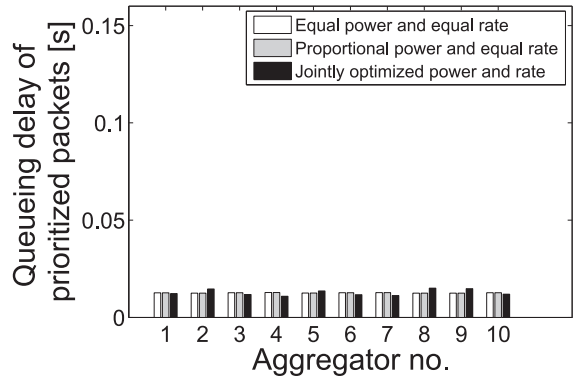

(c)

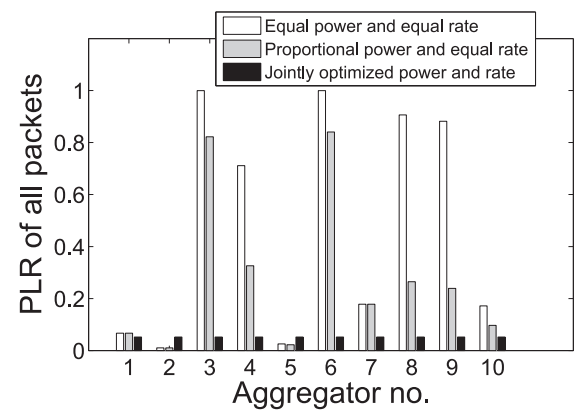

(b)

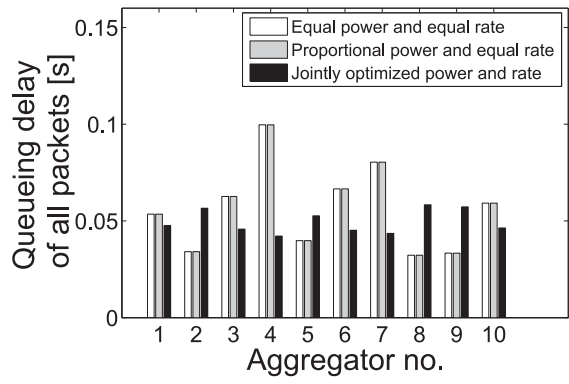

(d)

Fig. 8. Comparison of QoS metrics in an eHealth location with 10 subscribers: (a) PLR of prioritized packets, (b) PLR of all packets, (c) queuing delay of prioritized packets, and (d) queuing delay of all packets.

variation of path loss. The variation of the direction causes the variance of the received signal strength due to radio irregularity on different direction of propagation [32]. In order to simulate the variations of transmission power consumption caused by the variations of the distance and the direction, we change the transmission power consumption model at sensor $i$ to $P_{t, i}=\Omega \beta_{i} r_{i}$ where $\Omega$ is a Gaussian random variable with a variance of 0.01 and a mean of 1.0. The comparison of energy variation at sensor 5 between the static case and the dynamic case is shown in Fig. 6b.

There is a trade-off between the source rate and the lifetime of a sensor, as shown in Table 2. The uninterrupted lifetime of a sensor is the duration from the starting time of the sensor to the time when the energy level of the sensor reaches 0 at the first time. Table 2 shows that the uninterrupted lifetime of a sensor will be reduced as the source rate is increased. As shown in Table 2, when the source rate of sensor 3 is $847 \mathrm{bps}$, the uninterrupted lifetime of the sensor will be infinity. When the source rate of sensor 3 is increased to $1,695 \mathrm{bps}$, the uninterrupted lifetime of the sensor will be between 2,205 and 6,614 s.

We optimally allocate both the transmission power and the transmission rate at each aggregator to ensure that the data delivery from each aggreator meet the QoS require- 


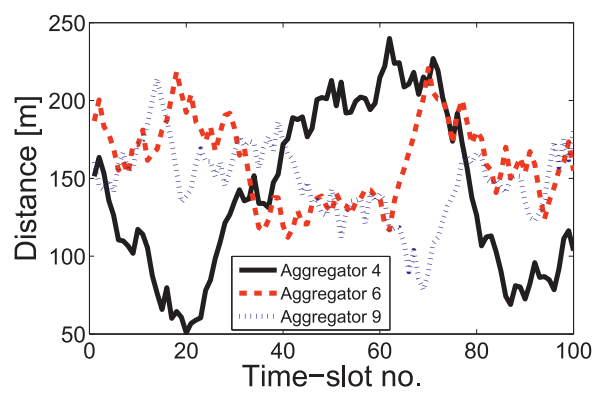

(a)

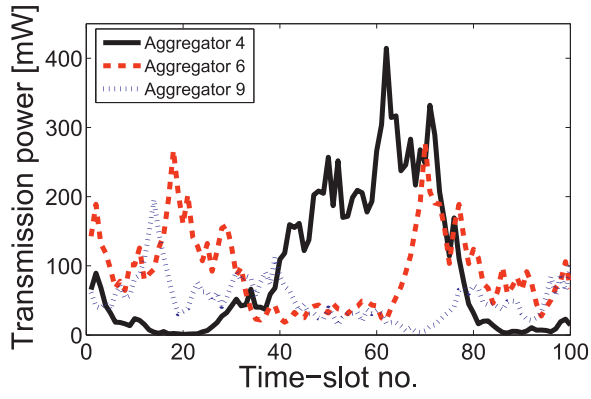

(b)

Fig. 9. Variation of transmission power due to the variation of the distance from the aggregator to the base station: (a) variation of the distance and (b) variation of the transmission power.

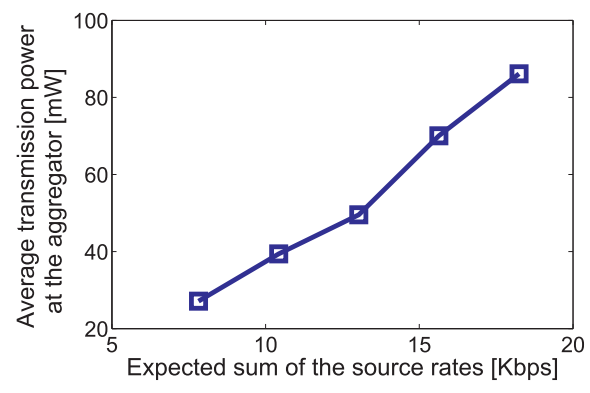

(a)

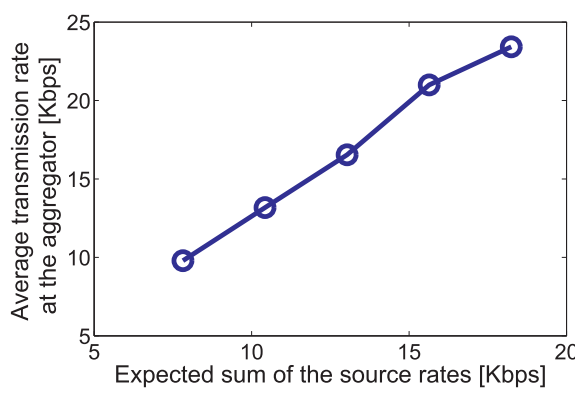

(b)

Fig. 10. Relationship between the source rates of the sensors in a BSN and the transmission power and the transmission rate at the aggregator: (a) average transmission power and (b) average transmission rate.

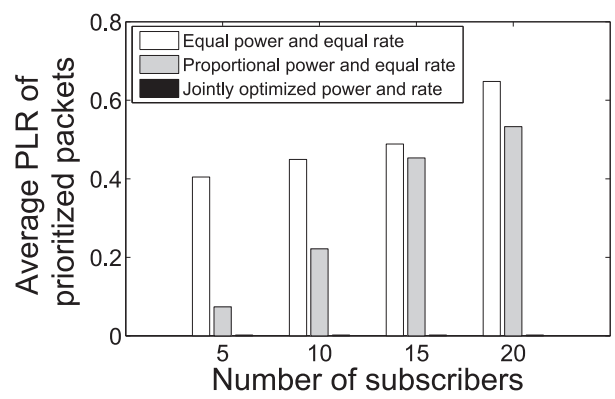

(a)

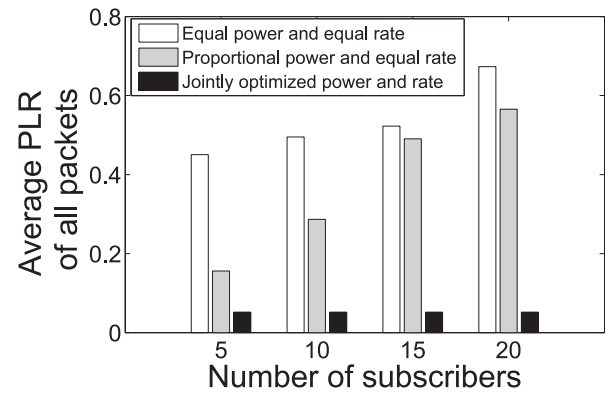

(b)

Fig. 11. Comparison of PLR with different number of subscribers in an eHealth location: (a) average PLR of prioritized packets and (b) average PLR of all packets.

ment. The optimal values of the transmission powers and the transmission rates, as shown in Fig. 7, are obtained by solving the optimization problem (16). From Fig. 7a, we see that the transmission powers vary greatly among different aggregators. The aggregators close to the base station (e.g., aggregators 1, 2, and 7) apply a lower transmission power than those far away from the base station (e.g., aggregators 3 and 6).

In Fig. 8, we compare the PLR and the delay of the prioritized packets and all the packets at the aggregator, respectively, among three schemes: 1) the scheme with Jointly Optimized Power and Rate (JOPR), which is the proposed solution to the optimization problem $(16), 2)$ the scheme with Equal Power and Equal Rate (EPER), in which the transmission powers and the transmission rates are equally allocated among the aggregators, and 3) the scheme with Proportional Power and Equal Rate (PPER), in which the transmission power at an aggregator is proportional to the distance from the aggregator to the base station, and the transmission rates are equally allocated among the aggregators. In order for fair comparison, the sum of the transmission powers and the sum of the transmission rates of all aggregators are equal among the three schemes. The proposed scheme optimizes the resources to provide QoS guarantee. The PLR for the prioritized packets at each aggregator is equal to 0.002, as shown in Fig. 8a, and the PLR for all the packets at each aggregator is equal to 0.052, as shown in Fig. 8b. The EPER scheme or PPER scheme does not allocate the resources appropriately, thus some far-way sensors (e.g., sensors 3 and 6) suffer from a larger PLR, as shown in Figs. $8 \mathrm{a}$ and $8 \mathrm{~b}$. From Figs. $8 \mathrm{c}$ and $8 \mathrm{~d}$, we can see that the difference of the queuing delays among the three schemes is very small. This is because the transmission rates, which determine the queuing delays, are close in 
the three schemes. At each aggregator, the prioritized packets are transmitted in priority, thus having a much smaller delay than the other packets.

In the pervasive health monitoring systems, the subscribers may move around even when they are being monitored. The transmission power at the aggregator needs to be optimized at each time slot taking into account the variation of the distance from the aggregator to the base station. Fig. 9 shows the variation of optimal transmission powers when the distances vary with time. The proposed algorithm optimizes the resource allocation using convex optimization, which enables a fast power adaptation at each aggregator.

The transmission power and the transmission rate at the aggregator are dependent on the source rates of the sensors in the BSN. As shown in Fig. 10, a higher average transmission power and a higher average transmission rate are required to provide the QoS guarantee if the sum of the source rates of the sensors is increased.

We vary the number of the subscribers from 5 to 20 in an eHealth location, and then compare the PLRs. The bandwidth is set to $500 \mathrm{Kbps}$ in the eHealth location. As shown in Fig. 11, the average PLRs of the prioritized packets and all the packets at the aggregator in the proposed JOPR scheme are 0.002 and 0.052 , respectively, much lower than those in the EPER scheme and the PPER scheme.

\section{Conclusions}

In this paper, we optimize the resource allocations to provide a sustainable and high-quality service in health monitoring systems. We first formulate and solve the steady-rate optimization problem, which optimizes the source rate at each sensor to minimize the rate fluctuation with respect to the average sustainable rate, subject to the requirement of uninterrupted service. Given the optimal source rates at each sensor, we then formulate and solve the QoS optimization problem, in which we jointly optimize the transmission power and the transmission rate at each aggregator to provide QoS guarantee to data delivery. The simulation results demonstrate that the optimal resource allocations improve the system performances in terms of sustainability and service quality in health monitoring systems.

\section{REFERENCES}

[1] K. Kinsella and V. Velkoff, An Aging World: 2001. US Census Bureau, 2001.

[2] O. Aziz, B. Lo, R. King, A. Darzi, and G.Z. Yang, "Pervasive Body Sensor Network: An Approach to Monitoring the Postoperative Surgical Patient," Proc. Int'l Workshop Wearable and Implantable Body Sensor Networks, pp. 15-18, Apr. 2006.

[3] A. Wood, J. Stankovic, G. Virone, L. Selavo, Z. He, Q. Cao, T. Doan, Y. Wu, L. Fang, and R. Stoleru, "Context-Aware Wireless Sensor Networks for Assisted Living and Residential Monitoring," IEEE Network, vol. 22, no. 4, pp. 26-33, July 2008.

[4] F. Chiti, R. Fantacci, F. Archetti, E. Messina, and D. Toscani, "An Integrated Communications Framework for Context Aware Continuous Monitoring with Body Sensor Networks," IEEE J. Selected Areas in Comm., vol. 27, no. 4, pp. 379-386, May 2009.

[5] D. Niyato, E. Hossain, and S. Camorlinga, "Remote Patient Monitoring Service Using Heterogeneous Wireless Access Networks: Architecture and Optimization," IEEE J. Selected Areas in Comm., vol. 27, no. 4, pp. 412-423, May 2009.
[6] G.Z. Yang, Body Sensor Networks. Springer, 2006.

[7] B. Lo and G.Z. Yang, "Body Sensor Network-Research Challenges and Opportunities," Proc. IET Seminar on Antennas and Propagation for Body-Centric Wireless Comm., pp. 26-32, Apr. 2007.

[8] A. Seyedi and B. Sikdar, "Modeling and Analysis of Energy Harvesting Nodes in Body Sensor Networks," Proc. Int'l Workshop Wearable and Implantable Body Sensor Networks, pp. 175-178, June 2008.

[9] A. Seyedi and B. Sikdar, "Energy Efficient Transmission Strategies for Body Sensor Networks with Energy Harvesting," Proc. Conf. Information Sciences and Systems (CISS '08), pp. 704709, Mar. 2008.

[10] A. Kansal, J. Hsu, S. Zahedi, and M.B. Srivastava, "Power Management in Energy Harvesting Sensor Networks," ACM Trans. Embedded Computing Systems, vol. 6, no. 4, Sept. 2007.

[11] G. Zhou, J. Liu, C. Wan, M. Yarvis, and J. Stankovic, "BodyQoS: Adaptive and Radio-Agnostic QoS for Body Sensor Networks," Proc. IEEE INFOCOM, pp. 565-573, Apr. 2008.

[12] B. Otal, L. Alonso, and C. Verikoukis, "Highly Reliable EnergySaving MAC for Wireless Body Sensor Networks in Healthcare Systems," IEEE J. Selected Areas in Comm., vol. 27, no. 4, pp. 553565, May 2009.

[13] W. Yu and J. Yuan, "Joint Source Coding, Routing and Resource Allocation for Wireless Sensor Networks," Proc. IEEE Int'l Conf. Comm. (ICC '05), pp. 737-741, May 2005.

[14] T. Shu, M. Krunz, and S. Vrudhula, "Joint Optimization of Transmit Power-Time and Bit Energy Efficiency in CDMA Wireless Sensor Networks," IEEE Trans. Wireless Comm., vol. 5, no. 11, pp. 3109-3118, Nov. 2006.

[15] A. Milenkovic, C. Otto, and E. Jovanov, "Wireless Sensor Networks for Personal Health Monitoring: Issues and an Implementation," Computer Comm., vol. 29, no. 13, pp. 25212533, 2006.

[16] R. Istepanian, N. Philip, and M. Martini, "Medical QoS Provision Based on Reinforcement Learning in Ultrasound Streaming over 3.5G Wireless Systems," IEEE J. Selected Areas in Comm., vol. 27, no. 4, pp. 566-574, May 2009.

[17] W. Seah, Z. Eu, and H. Tan, "Wireless Sensor Networks Powered by Ambient Energy Harvesting (WSN-HEAP)-Survey and Challenges," Proc. First Int'l Conf. Wireless Comm. Vehicular Technology, Information Theory and Aerospace and Electronics Systems Technology (Wireless VITAE '09), pp. 1-5, May 2009.

[18] K. Fan, Z. Zheng, and P. Sinha, "Steady and Fair Rate Allocation for Rechargeable Sensors in Perpetual Sensor Networks," Proc. Sixth ACM Conf. Embedded Network Sensor Systems (SenSys '08), pp. 239-252, Nov. 2008.

[19] M. Chiang, S.H. Low, A.R. Calderbank, and J.C. Doyle, "Layering as Optimization Decomposition: A Mathematical Theory of Network Architectures," Proc. IEEE, vol. 95, no. 1, pp. 255-312, Jan. 2007

[20] M. Chiang, C.W. Tan, D.P. Palomar, D. O'Neill, and D. Julian, "Power Control by Geometric Programming," IEEE Trans. Wireless Comm., vol. 6, no. 7, pp. 2640-2651, July 2007.

[21] H. Ren and M. Meng, "A Game Theoretic Model of Distributed Power Control for Body Sensor Networks to Reduce Bioeffects," Proc. IEEE/EMBS Int'l Summer School on Medical Devices and Biosensors, pp. 90-93, Sept. 2006.

[22] L. Kleinrock and F.A. Tobagi, "Carrier Sense Multiple-Access Modes and Their Throughput-Delay Characteristics," IEEE Trans. Comm., vol. 23, no. 12, pp. 1400-1416, Dec. 1983.

[23] R.D. Yates and D.J. Goodman, Probability and Stochastic Processes, a Friendly Introduction for Electrical and Computer Engineering, second ed. John Wiley \& Sons, Inc., 2004.

[24] W. Heinzelman, A. Chandrakasan, and H. Balakrishnan, "An Application-Specific Protocol Architecture for Wireless Microsensor Networks," IEEE Trans. Wireless Comm., vol. 1, no. 4, pp. 660670, Oct. 2002.

[25] T.S. Rappaport, Wireless Communications: Principles and Practice, second ed. Prentice Hall, 2002.

[26] J. Zhu, S. Chen, B. Bensaou, and K. Hung, "Tradeoff between Lifetime and Rate Allocation in Wireless Sensor Networks: A Cross Layer Approach," Proc. IEEE INFOCOM, pp. 267-275, 2007.

[27] N.U. Prabhu, Foundations of Queueing Theory. Springer Press, 1997.

[28] S. Boyd, S.-J. Kim, L. Vandenberghe, and A. Hassibi, "A Tutorial on Geometric Programming," Optimization and Eng., vol. 8, no. 1, pp. 67-127, Apr. 2007. 
[29] S. Boyd and L. Vandenberghe, Convex Optimization. Cambridge Univ., 2004.

[30] IEEE 802.15.4, Wireless Medium Access Control (MAC) and Physical Layer (PHY) Specifications for Low-Rate Wireless Personal Area Networks (LR-WPANs), IEEE, Oct. 2003.

[31] M. Kohvakka, M. Kuorilehto, M. Hannikainen, and T.D. Hamdainen, "Performance Analysis of IEEE 802.15.4 and Zigbee for Large-Scale Wireless Sensor Network Applications," Proc. ACM Int'l Workshop Performance Evaluation of Wireless Ad Hoc, Sensor and Ubiquitous Networks, pp. 48-57, Oct. 2006.

[32] G. Zhou, T. He, S. Krishnamurthy, and J.A. Stankovic, "Models and Solutions for Radio Irregularity in Wireless Sensor Networks," ACM Trans. Sensor Networks, vol. 2, no. 2, pp. 221-262, May 2006

[33] Y. Zhao, A. Sani, Y. Hao, S.L. Lee, and G.Z. Yang, "A Simulation Environment for Subject-Specific Radio Channel Modeling in Wireless Body Sensor Networks," Proc. Int'l Workshop Wearable and Implantable Body Sensor Networks, pp. 23-28, June 2009.

[34] S.L. Chen, H.Y. Lee, C.A. Chen, H.Y. Huang, and C.H. Luo, "Wireless Body Sensor Network with Adaptive Low-Power Design for Biometrics and Healthcare Applications," IEEE Systems J., vol. 3, no. 4, pp. 398-409, Dec. 2009.

[35] C.M. Vigorito, D. Ganesan, and A.G. Barto, "Adaptive Control of Duty Cycling in Energy-Harvesting Wireless Sensor Networks," Proc. IEEE Fourth Ann. Comm. Soc. Conf. Sensor, Mesh and Ad Hoc Comm. and Networks (SECON '07), pp. 21-30, June 2007.

[36] J. Hsu, S. Zahedi, A. Kansal, M. Srivastava, and V. Raghunathan, "Adaptive Duty Cycling for Energy Harvesting Systems," Proc. Int'l Symp. Low Power Electronics and Design (ISLPED '06), pp. 180185, Oct. 2006.

[37] C. Moser, L. Thiele, D. Brunelli, and L. Benini, "Adaptive Power Management for Environmentally Powered Systems," IEEE Trans. Computers, vol. 59, no. 4, pp. 478-491, Apr. 2010.

[38] J. Penders, B. Grundlehner, R. Vullers, and B. Gyselinckx, "Potential and Challenges of Body Area Networks for Affective Human Computer Interaction," Proc. Int'l Conf. Augmented Cognition, pp. 202-211, July 2009.

[39] J.A. Paradiso, "Systems for Human-Powered Mobile Computing," Proc. IEEE Design Automation Conf. (DAC '09), pp. 645-650, Sept. 2009.

[40] H. Su and X. Zhang, "Battery-Dynamics Driven TDMA MAC Protocols for Wireless Body-Area Monitoring Networks in Healthcare Applications," IEEE J. Selected Areas in Comm., vol. 27, no. 4, pp. 424-434, May 2009.

[41] ZigBee Specification, ZigBee Alliance, Dec. 2006.

[42] A. Olivares, G. Olivares, P. Gloesekoetter, J.M. Gorriz, and J. Ramirez, "A Study of VibrationBased Energy Harvesting in Activities of Daily Living," Proc. Int'l Conf. Pervasive Computing Technologies for Healthcare, pp. 1-4, Mar. 2010.

[43] Y.K. Huang, A.C. Pang, and H.N. Hung, "An Adaptive GTS Allocation Scheme for IEEE 802.15.4," IEEE Trans. Parallel and

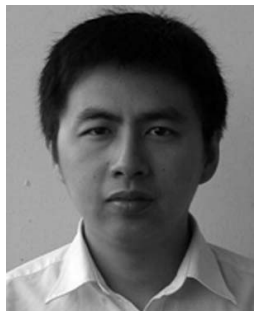
Distributed Systems, vol. 19, no. 5, pp. 641651, May 2008.

Yifeng He received the PhD degree in electrical engineering from Ryerson University, Canada, in 2008. He is currently an assistant professor in the Department of Electrical and Computer Engineering at Ryerson University. His research interests include body sensor networks, wireless visual sensor networks, peer-to-peer video streaming, and distributed optimizations for multimedia communications. $\mathrm{He}$ is the recipient of the 2008 Governor General's Gold Medal in Canada and the 2007 Pacific-Rim Conference on Multimedia (PCM) best paper award. He is a member of the IEEE.

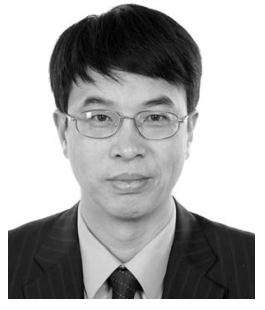

Wenwu Zhu received the BE and ME degrees from the National University of Science and Technology, China, in 1985 and 1988, respectively, the MS degree from the Illinois Institute of Technology, Chicago, and the PhD degree from the Polytechnic Institute of New York University in 1993 and 1996, respectively, all in electrical engineering. $\mathrm{He}$ is a senior researcher with the Internet Media Computing Group at Microsoft Research Asia. Prior to his current post, he was the director and chief scientist at the Intel Communication Technology Lab China as well as an Intel principal engineer from September 2004 to 2008. He previously served with Microsoft Research Asia in the Internet Media Group and Wireless and Networking Group as a research manager from October 1999 to August 2004. He worked for Bell Labs as a member of the technical staff during 1996-1999. He has published more than 200 refereed papers in multimedia communications and networking and wireless communications. He participated in the IETF $\mathrm{ROHC}$ WG on robust TCP/IP header compression over wireless links and IEEE $802.16 \mathrm{~m}$ WG standardization. He is the inventor or coinventor of more than 40 patents. His current research interest is in the area of wireless/Internet multimedia communication and networking. He has severed on various editorial boards of IEEE journals, such as guest editor for the Proceedings of the IEEE and IEEE JSAC and associate editor for the IEEE Transactions on Mobile Computing, the IEEE Transactions on Multimedia, and the IEEE Transactions on Circuits and Systems for Video Technology. He received the Best Paper Award from the IEEE Transactions on Circuits and Systems for Video Technology in 2001 and the Best Paper Award in 2004 from the Multimedia Communication Technical Committee of the IEEE Communications Society. He is a past chair of the Visual Signal Processing and Communication Technical Committee of IEEE Circuits and Systems Society. He currently serves as the chairman of the IEEE Circuits and Systems Society Beijing Chapter, on the steering committee of the IEEE Transactions on Mobile Computing, and on the advisory board of the International Journal of Handheld Computing Research. He will serve as the TPC chair for IEEE ISCAS 2013, Beijing. He is a fellow of the IEEE.

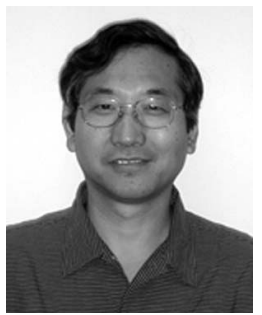

Ling Guan received the bachelor's degree from Tianjin University, China, the master's degree from the University of Waterloo, and the PhD degree from the University of British Columbia. He is a Tier I Canada Research chair in Multimedia and Computer Technology and a professor of electrical and computer engineering at Ryerson University, Toronto, Canada. He has been working on image, video, and multimedia signal processing and has published extensively in the field. He currently serves on the editorial boards of the IEEE Transactions on Circuits and Systems for Video Technology and numerous other international journals. $\mathrm{He}$ chaired the 2006 IEEE International Conference on Multimedia and Expo in Toronto, cochaired the 2008 ACM International Conference on Image and Video Retrieval in Niagara Falls, and served as the founding general chair of IEEE Pacific-Rim Conference on Multimedia in 2000. He is an IEEE Circuits and System Society Distinguished lecturer (2010-2011) and a recipient of the 2005 IEEE Transactions on Circuits and Systems for Video Technology Best Paper Award. He is a fellow of the IEEE, a fellow of the Engineering Institute of Canada, and a fellow (elected) of the Canadian Academy of Engineering.

$\triangle$ For more information on this or any other computing topic, please visit our Digital Library at www.computer.org/publications/dlib. 